Gorges, C.; Öztürk, K.; Liebich, R.

\title{
Impact detection using a machine learning approach and experimental road roughness classification
}

Journal article | Accepted manuscript (Postprint)

This version is available at https://doi.org/10.14279/depositonce-8145

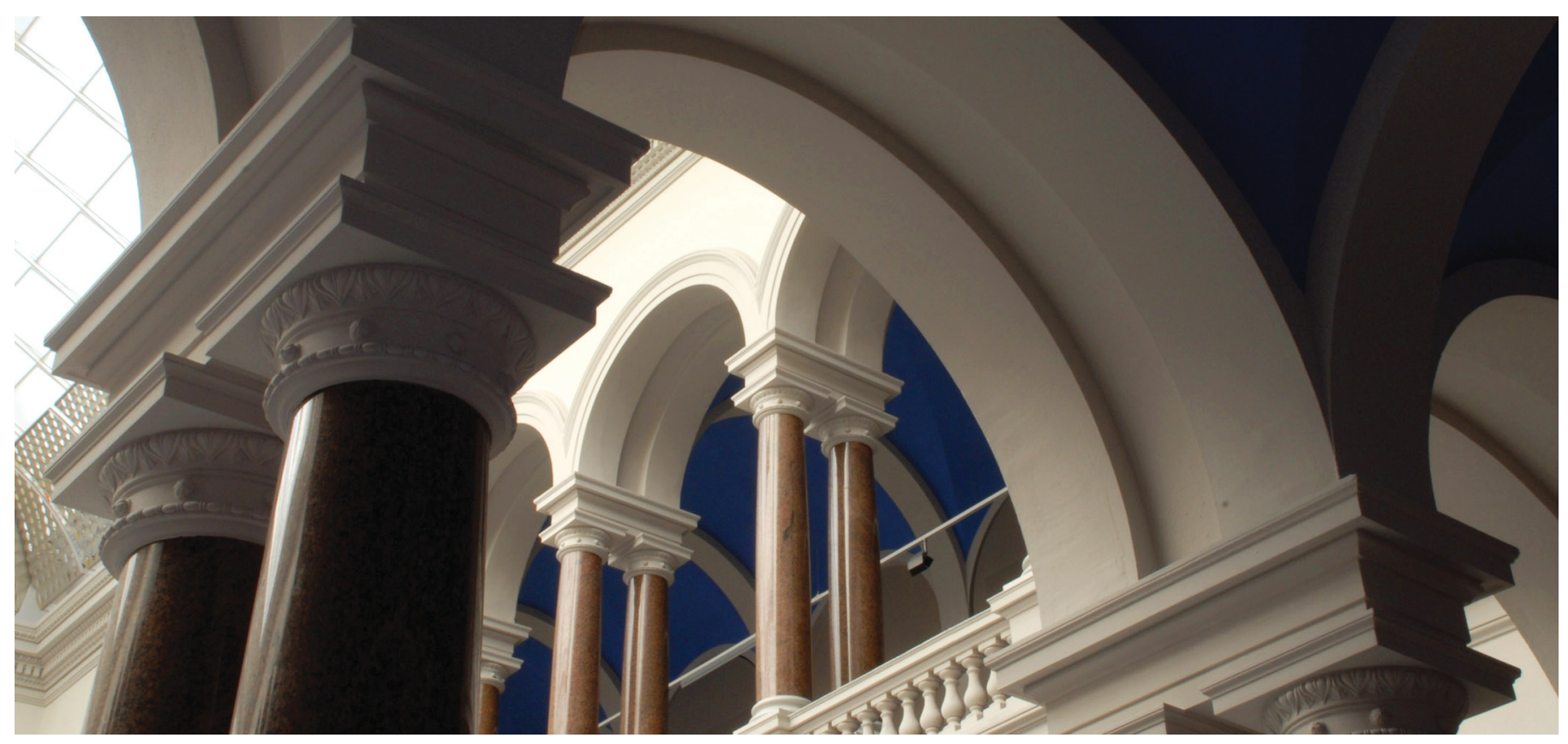

Gorges, C., Öztürk, K., \& Liebich, R. (2019). Impact detection using a machine learning approach and experimental road roughness classification. Mechanical Systems and Signal Processing, 117, 738-756. https://doi.org/10.1016/j.ymssp.2018.07.043 


\title{
Impact detection using a machine learning approach and experimental road roughness classification
}

\author{
Christian Gorges ${ }^{\mathrm{a}}$, Kemal Öztürk ${ }^{\mathrm{a}}$, Robert Liebich ${ }^{\mathrm{b}}$ \\ ${ }^{\mathrm{a}}$ BMW AG, Petuelring 130, 80788 Munich, Germany $;{ }^{\mathrm{b}}$ Chair of Engineering Design \\ and Product Reliability, Berlin Institute of Technology, Straße des 17. Juni 135, 10623 \\ Berlin, Germany
}

(This is an Accepted Manuscript of an article published by Elsevier in Mechanical Systems and Signal
Processing on 15/02/2019, available online: https:// doi.org/10.1016/j.ymssp. 2018. 07. 043.)

First, this publication presents the experimental validation of a road roughness classification method. Second, an impact detection strategy for twowheeled vehicles is proposed including a classification of service loads, mild special events, and severe special events. The methods presented utilise the vehicle's onboard signals to gather field data. The modular road roughness classification system operates with the vehicle's transfer functions, and continuously classifies the road profile, according to ISO 8608. The method was successfully validated on test tracks with known road profiles. The impact detection strategy was developed using a supervised machine learning technique. Six road obstacles were ridden over using different velocities to invoke mild and severe special events. The most popular classifiers were trained for comparison and prediction of future observations. The developed impact detection strategy shows a high accuracy and was successfully validated using a $\mathrm{k}$-fold cross-validation. The combination of the road roughness classification system and the impact detection strategy, enables a holistic field data acquisition of customer usage profiles, in the context of durability engineering. The collection of customer usage profiles improves vehicle design targets and enables a virtual load acquisition.

Keywords: Road roughness classification, ISO 8608, impact detection, supervised machine learning, customer usage profiles, two-wheeled vehicles

\section{Introduction}

The present publication combines a road roughness classification method with an impact detection strategy, in the context of durability engineering. Both methods are presented as an application for two-wheeled vehicles and were validated by experiments. The estimation and evaluation of the actual road roughness with the vehicle's onboard signals, has been the focus of many publications. The information about road roughness is often applied for active suspension systems or road surface maintenance. In the present research, the current road roughness is revealed and classified, to obtain a distribution 
of driven road classes, in the sense of field data collection. The underlying method of estimating the road roughness was developed by Gorges et al. [1] and is experimentally validated in the present publication. The modular road roughness classification system utilises the vehicle's transfer functions and classifies the road profile according to ISO 8608 [2]. A measurement campaign was carried out, which included rough, unpaved roads and obstacles for the experimental validation. The methods have been tested and validated with a real motorcycle ridden on test tracks, which were surveyed by a 3D roughness measurement system. The collection of driven road classes is part of gathering customer usage profiles, which improve vehicle design targets, especially in terms of lightweight design. Furthermore, customer usage profiles enable a virtual load acquisition on virtual test tracks. Thus, the knowledge about driven road classes is an improvement in the product development.

In the context of durability, the combination of customer behaviour and road roughness indicates whether the loads that occur are considered as service loads, special events, or even misuse events, as Figure 1 shows. According to the authors, an increase in the vehicle velocity induces higher loads for a given road roughness, and vice versa, see Figure 1a. Especially in the automotive industry, and referring to Matz [3] and Pötter [4], customer loads are divided in three categories: service loads, special events, and misuse events, as shown in Figure 1b. On the one hand, this separation is characterised by statistical considerations. On the other hand, this distinction is necessary for product liability and warranty. For example, an off-road motorcycle is designed for unpaved roads and even small jumps, which means, up to a specific threshold, the impacts of these events are regarded as service loads. While for a superbike, the manoeuvres described would be regarded as special events or misuse events. Johannesson and Speckert [5] described the customer load distribution "in terms of vehicle-independent load environment together with the vehicle usage and the vehicle dynamics". This description coincides with the presented derivation of customer loads.

Service loads occur during the normal use of the vehicle, which is often called the intended purpose. They can be described by a continuously distributed load spectra during the life of the vehicle. In the case of a motorcycle, service loads comprise acceleration and brake manoeuvres, cornering, and loads that occur due to the roughness of the road surface. In addition to the service loads, the intended purpose also includes the occurrence of special events. Special events are rare compared to service loads, and they induce a higher load on the vehicle components. They are often characterised by impacts from sudden events, for example, driving over a pothole. As special events are part of the intended use of the vehicle, the components must be designed to sustain the loads. 


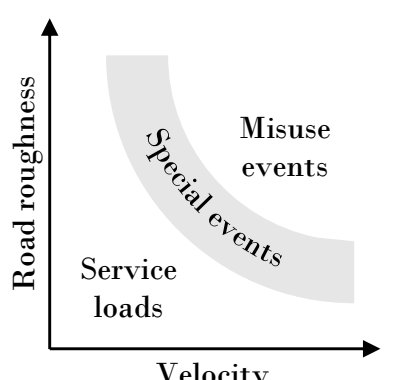

(a) as a combination of road roughness and vehicle velocity

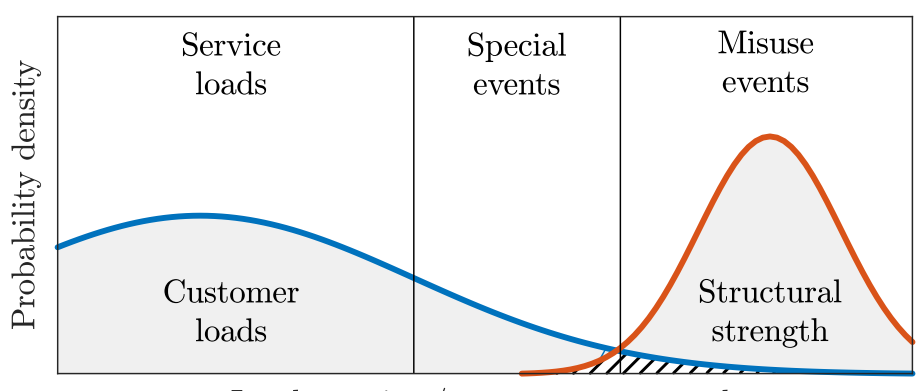

Load severity / component strength

(b) as a probability distribution

Figure 1 - Classification of customer loads.

After the occurrence of a special event, the vehicle still has to be fully operational.

Misuse events are per definition not part of the intended purpose, but they are also considered during the vehicle design process. In engineering, the fail-safe principle is applied, which means that the components should deform plastically along the load path. This is called the damage chain. Figure $1 b$ shows that in misuse events, the load severity typically coincides with the structural strength of the components. The customer should be able to clearly identify the damaged structure, and recognise that the components were over-exposed in consequence of the misuse event. Misuse events are characterised by a higher load level that exceeds a defined threshold, and are also often the consequence of impacts, for example riding against, or over a significant obstacle. The load threshold between special events and misuse events, is often determined by numerical simulation and validated with experiments. Further aspects of misuse, in the context of structural durability, have been discussed by Köhler et al. [6], Hauke [7], and Berger et al. [8]. The methods presented can be categorised as condition monitoring systems. For two-wheeled vehicles, the publication of Gorges et al. [9] shows a real-time, wheel force calculation, with subsequent rainflow counting, to derive customer loads. An example for passenger cars can be found in Matz [3].

The road roughness classification method presented, is designed for a continuous evaluation of the driven road classes. Due to the restrictions of the underlying full-vehicle model, the method works under normal operation conditions, which means service loads. The system is not intended for the detection of single events, for example passing over obstacles, nor for the evaluation of the loads that occur. For this reason, the second part of the present study investigates the development of an impact detection strategy. Since the threshold between service loads, special events, and misuse events is, not defined by 
specific loads and strains, a machine learning approach was evaluated. Different road obstacles were ridden over, at different velocities, to gather an adequate data set of labelled observations. Subsequently, various supervised machine learning techniques, in form of classification, were evaluated. It is called supervised machine learning, because the data set was labelled before the classifier was trained. Knowledge about the distribution of special events during the product's life, is highly valuable for improving vehicle design targets.

This paper is organised as follows: Section 2 describes the measurement and evaluation of longitudinal road profiles. Section 3 presents the experimental set-up and the test tracks for the measurement campaign. Section 4 briefly explains the methodology for the road roughness classification, and shows the adjustments for real operation conditions. The impact detection strategy is presented in Section 5. Finally, Section 6 shows the results of the study. Section 7 provides the publication's findings.

\subsection{Literature review for road roughness classification}

As mentioned above, information about the road roughness has various applications. González et al. [10], Harris et al. [11], and Ngwangwa et al. [12] presented methods for estimating road roughness in the context of road maintenance. Furthermore, the evaluation of the current road roughness makes active suspension systems possible, as shown in [13-24]. Burger [25] and Fauriat et al. [26] developed methods of deriving customer usage profiles in terms of durability, as was also the focus of Gorges et al. [1]. Different techniques were utilised to estimate the road roughness. For example, Ngwangwa et al. [12] and Yousefzadeh et al. [27] applied an Artificial Neuronal Network (ANN) to reconstruct and classify the road profile, depending on the measured vehicle responses. ANNs usually require high computational efforts for an online application and a large set of training data. Recently, Qin et al. [21] utilised deep learning techniques for classifying the road profile. Sliding mode observers were developed by Imine et al. [28] and Rath et al. [29]. More examples of the application of control theory can be found in Doumiati et al. [15, 16] and Tudón-Martínez et al. [18]. The control theory methods cited require more signals than the present set-up can provide. The application of Kalman filters was investigated by Doumiati et al. [13], Yu et al. [14], Jeong et al. [30], Fauriat et al. [26], Wang et al. [19], and Qin et al. [22]. Tudón-Martínez et al. [17] also examined an $\mathscr{H}_{\infty}$ observer to estimate the road roughness. An inverse control problem was formulated, and solved, by Burger [25] to estimate the road profile with the help of the control-constraints method, which requires the solution of differential-algebraic equations. Other mathematical optimisation techniques were applied by Harris et al. [11] 
and Nordberg [31]. An application of wavelet transformation was developed by Qin et al. [23, 32, 33] and Solhmirzaei et al. [34]. Ben Hassen et al. [24] utilised the Independent Component Analysis (ICA) for estimating the road profile with the responses of a fullvehicle model. The rather simple, but fast, approach of estimating the road profile in the frequency domain with the help of transfer functions, has been published by González et al. [10] and Barbosa [35-37]. This approach was also utilised by Gorges et al. [1] and extended to a full-vehicle model with a delayed real-wheel excitation. The novel contributions of this research were a sliding window with a small time span and velocitydependant transfer functions. The proposed method of road roughness classification was validated with a full-vehicle model and a numerical simulation. Since the study showed quite promising results, the method has now been extended to real working conditions, and validated with the help of experiments from the measurement campaign.

\subsection{Literature review for the detection of road irregularities}

A lot of road condition monitoring systems already exist. For example, De Zoysa et al. [38] developed a system for public transport, to monitor road deterioration in third world countries. They used a direct correlation of acceleration signals to the road surface condition. Another example of crowd-based monitoring systems, is the Pothole Patrol $\left(\mathrm{P}^{2}\right)$ [39], which makes use of the GPS and vibration sensors mounted on taxis to monitor the civil infrastructure in Boston. They use a threshold detection $z_{-}$peak to detect potholes, and a speed vs. z_ratio filter, to deal with the velocity dependency of the signals. The system was successfully validated to detect potholes and other severe road anomalies. The same technique is used by the Nericell and TrafficSense project, published by Mohan et al. [40]. Perttunen et al. [41] used a FFT transformation of the acceleration signals to extract frequency domain features and a method of linear regression to remove the speed dependency. Tai et al. [42] developed a smartphone-based road anomaly detector, especially for motorcycles. They used machine learning techniques to train a classifier. Mednis et al. [43] developed an Android based smartphone application as a layer, for the existing navigation system Waze. They also utilised the acceleration signals collected from the smartphones, to detect irregularities. Further examples of crowdsourced pothole detection systems that utilise smartphone sensors, are the Streetbump project from Carrera et al. [44], bump detection from Hoffmann et al. [45], S-Road Assist from Sharma et al. [46], Pothole detection from Wang et al. [47], RoADS from Seraj et al. [48], or the work of P.M. and Gopi [49], who used Gaussian model-based mining to detect abnormal events. A recent work from Fox et al. [50] shows the development of a multi-lane pothole detector, using accelerometer data from embedded vehicle sensors. 
They used Support Vector Machines (SVM) as machine learning technique. The research of Cong et al. [51] shows the application of wavelet packet decomposition for feature extraction of acceleration signals to detect road anomalies. A one-class SVM was used as a classifier. A more sophisticated approach was carried out by Li et al. [52]. The authors developed a model-based pothole detection application, which exploits a multiphase dynamic model of the tyre. A Bayesian estimation and an Unscented Kalman Filter (UKF) estimate the current mode. The angular wheel velocity, vehicle velocity, and vertical acceleration are provided as inputs.

All of the projects mentioned can be categorised as response-type detection systems, since they utilise the acceleration signal from either the vehicle itself, or from an additionallymounted embedded system, or smartphone. As well as these, vision-based systems have evolved due to the increase in advanced driver assistance systems, which include stereo cameras and Radar or Lidar sensors. Some examples of ultrasonic applications, are the pothole detection systems from Hedge et al. [53] and Madli et al. [54]. Examples of stereo vision based applications for pothole detection, can be found in [55-59]. A Lidar application can be found in [60]. The related work makes significant efforts to detect potholes and other severe road anomalies, in the sense of road surface condition monitoring. Therefore, the velocity dependency in the response signals has been removed to identify the road irregularities, exactly as they occur on the road. From a durability point of view, whether a vehicle drives over a pothole with a low or high velocity, is of major importance. Hence, the impact generated is dependant on both the obstacle itself and the velocity. In most cases, the velocity of the vehicle decides whether the manoeuvre is a service load, special event or even, misuse event. For this reason, the authors decided not to develop a pothole detection system, but an impact detection system instead. This system should be able to detect and classify special events, regardless of whether it was a pothole, manhole, speed bump, or kerb. Due to the stochastic nature of special events, the detection strategy has been chosen to be an event detection in the time domain. With the help of measurements of such special events, an impact detection strategy was developed.

\section{Longitudinal road profiles}

A definition and illustration of longitudinal road profiles can be found in the previous research by Gorges et al. [1]. Longitudinal road profiles are defined as slices of the road surface in the direction of the road. In contrast, lateral road profiles describe the roughness and texture of the road, as a function of the track width. This is important 
for two-track vehicles but is ignored in the case of single-track vehicles. The influence of driving manoeuvres is neglected in the present research, and the longitudinal road profile is assumed to be the driven road profile. For the majority of the distance driven, this assumption appears to be justified. Furthermore, this simplification is necessary for the validation of the road roughness classification system with the measured test tracks.

\subsection{Road profilers}

Road profilers or profilometers, measure the texture of road surfaces. They exist in several variations, but all require three key elements: a reference elevation, a height relative to the elevation, and a longitudinal distance [61]. General Motors Research Laboratories [62] developed the first inertial profilers in 1960, to measure large road networks at high speed. The inertial reference is measured by an accelerometer and the relative height is measured by a non-contacting sensor, e.g. a laser transducer. The measurement of road roughness with inertial profilers requires a minimum speed. These methods have been proven to produce accurate results, even if they cannot collect long road undulations. However, in terms of durability, spatial frequencies less than $n=0.01$ cycles $/ \mathrm{m}$ (wavelengths above $100 \mathrm{~m}$ ) are negligible [61]. Further road profilers have been developed, for instance, the Motor Industry Research Association (MIRA) device [63] or the Longitudinal Profile Analyzer [28], where a car tows a single-wheel trailer and the movement of the wheel is transformed into the profile elevation. Barbosa [37] developed a similar road profiler, whereby the wheel movement is transformed using the systems transfer function to calculate the road profile. Once the road profiles are collected as a function of the distance, they can be evaluated with different techniques. In this study, a mobile, multi-sensor, measuring system from 3D Mapping Solutions $\mathrm{GmbH}^{1}$ has been utilised to survey the test tracks. High-performance laser scanners sample the road surface in three dimensions with a precision $<1 \mathrm{~mm}$. The scanned surface is provided in the OpenCRG® ${ }^{2}$-format. It is an open standard file format for the evaluation of high-precision microscopic road surface data, for analysing handling, ride comfort, and durability. Further information about the file format can be found in $[64,65]$. After the data had been processed and saved in the described format, a longitudinal road profile was extracted for a given vehicle trajectory. For the extraction of the road profile, a coarser sampling rate of $n=20$ cycles $/ \mathrm{m}$ was chosen, because the evaluation of the road profiles does not require a fine resolution, due to the enveloping

\footnotetext{
${ }^{1}$ 3D Mapping Solutions GmbH, Raiffeisenstrasse 16, D-83607 Holzkirchen, http://www . 3d-mapping. de

${ }^{2}$ Curved Regular Grid, http://www.opencrg.org
} 
effect of the tyres.

\subsection{Evaluation of longitudinal road profiles}

The evaluation of longitudinal road profiles was discussed in the previous research by Gorges et al. [1]. In summary, there are two common methods for evaluating the road roughness; the International Roughness Index (IRI) and the Power Spectral Density (PSD) approach. The IRI represents the road roughness on a single scale, whereas the PSD approach allows a classification of the road roughness into eight different road classes $(\mathrm{A}-\mathrm{H})$. In addition, the classification can be performed at different spatial frequencies. In general, the PSDs of road profiles are evaluated for standard road segment lengths; for example, $1 \mathrm{~km}$. The previous research by Gorges et al. [1] introduced a novel smoothing algorithm to handle PSDs even for small time spans, which is necessary for a fast detection of the current road roughness. The present research experimentally validates this methodology, which is reviewed briefly in Section 4. Examples of smoothed PSDs can be found in Section 3.2, at the evaluation of the test tracks.

\section{Measurement campaign}

\subsection{Experimental set-up}

A motorcycle (BMW R1200GS) was mounted with data-logging devices for experimental tests and validation of the algorithms. The following onboard signals were logged during the measurement campaign, for an offline simulation of the developed algorithms:

- vehicle velocity $v$,

- front and rear suspension travel $s_{\mathrm{ft}}, s_{\mathrm{rr}}$, and

- model-based signals (e.g. roll angle $\varphi$ ).

These signals were logged through the Controller Area Network (CAN) bus. Additionally, the vehicle was equipped with a Global Positioning System (GPS) logging device, which provided information about its position, for subsequent validation. The logged sig-


algorithms. The discrete model uses the same time step size as the vehicle's onboard system, which is set to $t_{\mathrm{s}}=0.01 \mathrm{~s}$. This, in principle, enables an online application of the developed algorithms. The evaluation of specific hardware requirements for an implementation of the methods into existing or new, production vehicles, is not part of the present study. 
Table 1 - Properties of test tracks.

\begin{tabular}{|c|c|c|c|c|c|}
\hline Test track No. & $\begin{array}{c}\text { ISO } \\
\text { Class }\end{array}$ & Description & $\begin{array}{c}\mathrm{L} \\
(\mathrm{m})\end{array}$ & $\begin{array}{c}\mathrm{IRI} \\
\left(\mathrm{m} \mathrm{km}^{-1}\right)\end{array}$ & $\begin{array}{l}\text { CRG } \\
(y / n)\end{array}$ \\
\hline$\# 1$ & A & High-speed test track & 7428 & 1.13 & $\mathrm{y}$ \\
\hline$\# 2$ & $\mathrm{~B}$ & Bumpy country road & 916 & 3.94 & $\mathrm{y}$ \\
\hline$\# 3$ & $\mathrm{~B}$ & Dilapidated country road & 720 & 4.21 & $\mathrm{y}$ \\
\hline$\# 4$ & B & Fine cobblestone & 266 & 5.40 & $\mathrm{y}$ \\
\hline$\# 5$ & $\mathrm{C}$ & Dilapidated concrete panels & 209 & 6.24 & $\mathrm{y}$ \\
\hline$\# 6$ & $\mathrm{C}$ & Coarse cobblestone & 495 & 7.97 & $\mathrm{y}$ \\
\hline$\# 7$ & - & Gravel road & 836 & - & $\mathrm{n}$ \\
\hline$\# 8$ & - & Rough unpaved roads & 6742 & - & $\mathrm{n}$ \\
\hline$\# 9$ & - & Enduro fun park & 12296 & - & $\mathrm{n}$ \\
\hline
\end{tabular}

\subsection{Test tracks for road roughness classification}

Nine different test tracks were ridden over to validate the road roughness classification algorithm. Table 1 shows the properties of the selected test tracks. Six of them were surveyed by the 3D roughness measurement system in a previous study by BMW, which are denoted by the Curved Regular Grid (CRG) column (y/n). The ISO classification result was achieved at the reference spatial frequency $n_{0}=0.1$ cycles $/ \mathrm{m}$, see Section 2.2 for the evaluation method. The IRI was calculated directly from the longitudinal road profile, whereas the ISO classification results from the profile's PSD. Test track No. 1, is a high-speed test track at BMW's test and performance centre, see Figure 2. The track is characterised by a very smooth surface, made for high velocity manoeuvres. The classification results are ISO class A and a low IRI, which means that very little suspension motion occurs while driving on the test track. Test track No. 2 is an artificial, bumpy, country road, also located at BMW's test and performance centre. It was constructed to test vehicles in terms of comfort and long-wave excitation. The profile was classified as ISO class B. Another example of an ISO class B road, is test track No. 3. This dilapidated country road is located in the Munich countryside. It is characterised by a long-wave excitation and a crumbled surface with some defects.

Test track No. 4 represents fine cobble stone. This road surface is characteristic for the historic centres of European cities, for example Milan or Rome, and excites the vehicle at very short wavelengths. It was also classified as ISO class B. An extract of test track No. 4's longitudinal road profile, is illustrated in Figure 3. It also shows the histogram of the road profile, together with a Gaussian distribution. The similarity between the probability distribution and the normal distribution, shows the stochastic nature of road profiles, as discussed in Section 2.2. An example of an ISO class C road, is test track No. 5. This test track is also located at the test and performance centre, and represents a bad highway made of dilapidated concrete panels. The connections between the concrete 


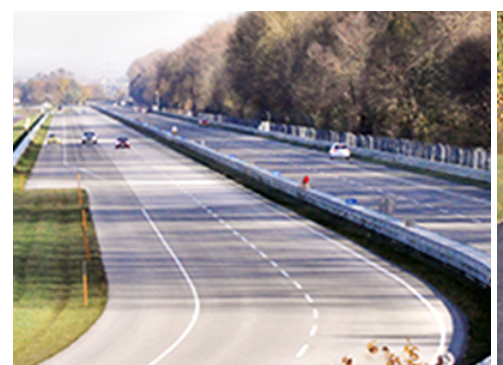

\#1: High-speed test track

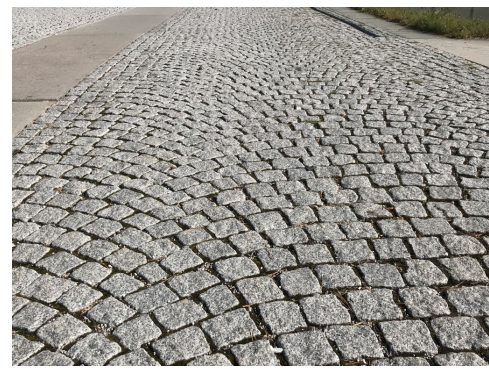

\#4: Fine cobblestone



\#7: Gravel road

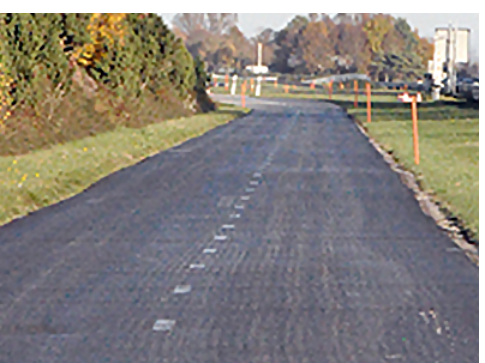

\#2: Bumpy country road

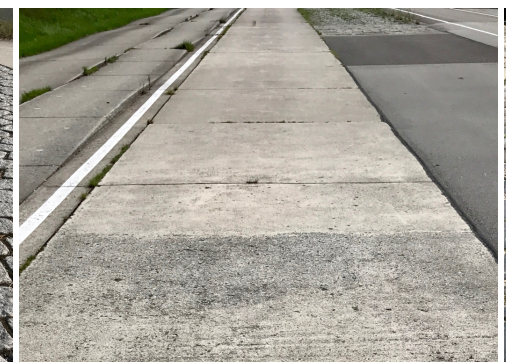

\#5: Dilapidated concrete panels

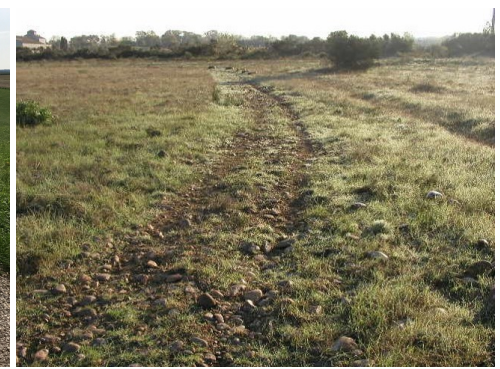

\#8: Rough unpaved roads

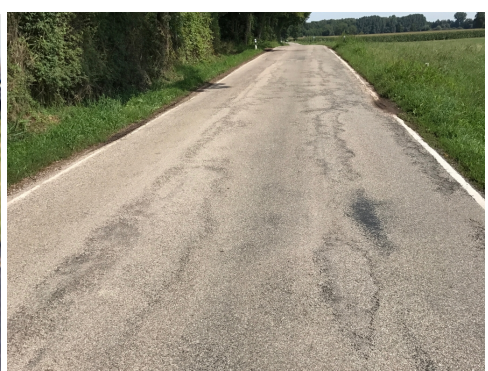

\#3: Dilapidated country road

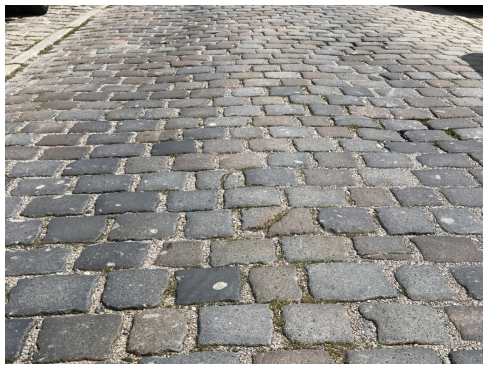

\#6: Coarse cobblestone

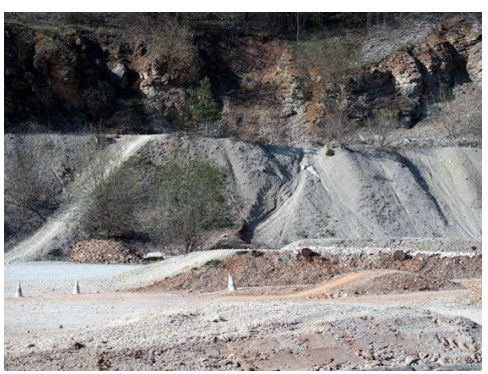

\#9: Enduro fun park

Figure 2 - Test tracks for the road roughness classification.

panels are rough, and this results in mid-wave excitation of the vehicle. This pavement is specific for older, dilapidated highways. Test track No. 6, which is part of the castle square at the Nymphenburg Palace in Munich, is made of coarse cobblestone. The road surface induces short-wave excitation. It was also classified as ISO class C.

Test track No. 7 consists of a gravel road and is located in the Munich countryside. It is a public road with some small defects. The gravel induces high-frequency excitation. Rough, unpaved roads are represented by test track No. 8, which is part of the test and performance centre. This test track consists of several natural and artificial dirt tracks, which were constructed for off-road motorcycle tests. These trails represent the tracks which are used during the increasingly popular enduro motorcycle adventure tours. The roughest test track within the measurement campaign, is test track No. 9. It is part 

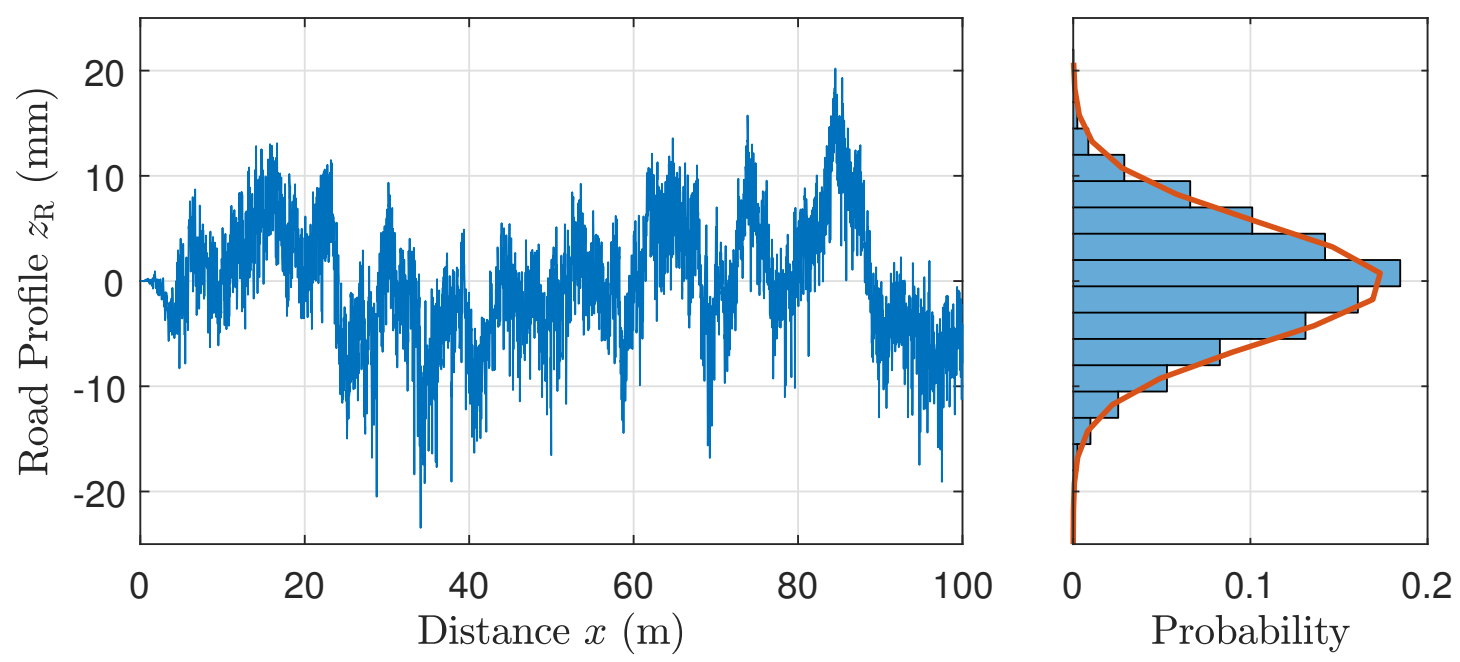

Figure 3 - Road profile and histogram of test track No. 4.

of the enduro fun park in Hechlingen, Germany, where special manoeuvres for off-road training are practised. These tracks are characterised by rough unpaved roads with large defects, potholes, and obstacles, for example, fallen tree trunks, roots, and stones. Test tracks No. 7-9 are ordered according to their subjectively perceived, roughness.

ISO classes of $\mathrm{C}$ and higher are characterised by unpaved roads, as the road surface gets more and more dilapidated with a higher roughness classification. As a consequence, these unpaved roads cannot be surveyed correctly. This is not a restriction of the measurement system but rather of the topology itself. On the one hand, the road surface of unpaved roads can change due to weather and wind. On the other hand, the vehicle itself displaces the loose underground as it rolls over it. This implies that the validation of the road roughness classification system for unpaved roads cannot be achieved by $3 \mathrm{D}$ roughness measurement. However, the authors propose to evaluate the unpaved roads presented, which have no measurement data available. The validation of these roads was achieved by a relative comparison between them, and also by comparing them to the surveyed roads.

Figure 4 shows the smoothed PSD of the test tracks No. 1, 2, 5, and 6, together with the ISO classification thresholds of the classes A-E. After the PSDs of the road profiles had been calculated, the smoothing algorithm computed one data point for each of the ten octave centre frequencies. The octave bands are highlighted with dashed lines. The reference spatial frequency $n_{0}=0.1$ cycles $/ \mathrm{m}$ is also highlighted with a solid line, at which the simple ISO classification is performed. According to this simplified classification procedure, the PSD at the other spatial frequencies, does not contribute 


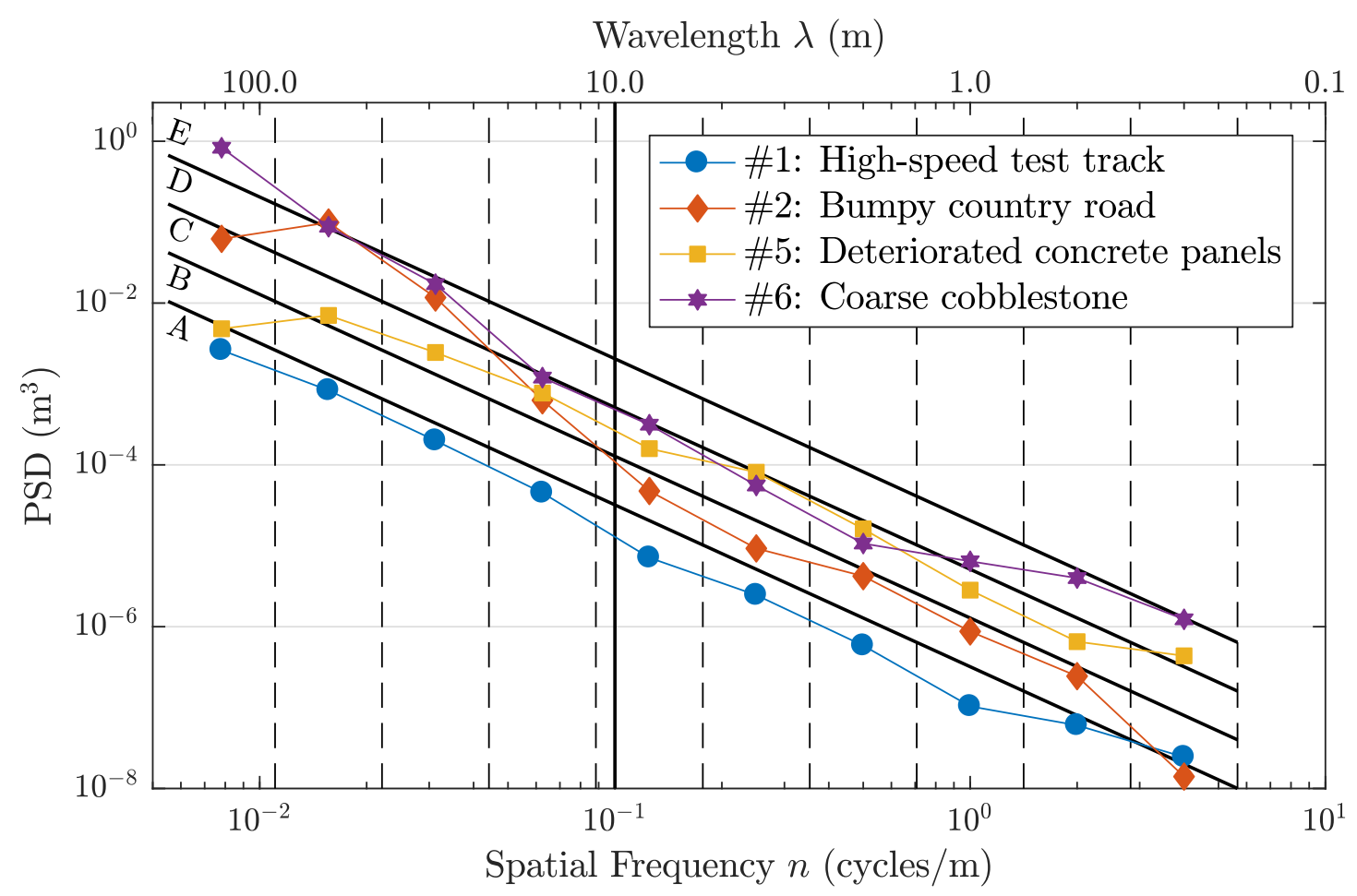

Figure 4 - Smoothed PSDs of selected test tracks.

to the classification result. This follows from the straight-line approximation of the PSD. On the contrary, the smoothed PSDs show that only test track No. 1 could be approximated by a straight line fit. Test track No. 5 also shows a more or less linear behaviour, excluding the first and last octave. The bumpy country road has a high degree of roughness at low spatial frequencies, with a decrease in roughness at the higher spatial frequencies. In contrast, test track No. 6 has a lower roughness at the mid spatial frequencies, compared to the roughness at the lower and higher spatial frequencies. As Andrén [66] also discovered, the straight-line approximation is not suitable for arbitrary test tracks.

In summary, a profile of a real, existing, road can be classified either by the simplified straight-line approximation, or in multiple octave bands at different spatial frequencies. In the case of the straight-line approximation, the PSD is evaluated solely at the reference spatial frequency. Other spatial frequencies are ignored and this results in an inaccurate classification result. On the other hand, a classification at multiple octave bands is also impractical, since this results in multiple classification results. Therefore, a more convenient classification method is proposed by the authors in the previous research [1]. Thus, a short road segment is classified by a minimum distance classifier into one of the 




\#1: Railroad crossing lateral

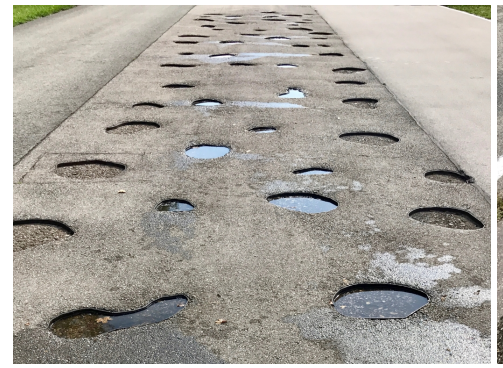

\#4: Pothole small

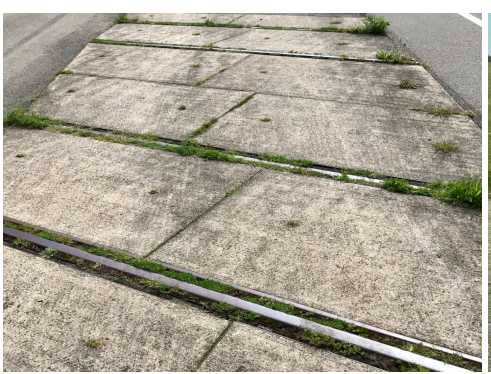

\#2: Railroad crossing angular



\#5: Pothole large

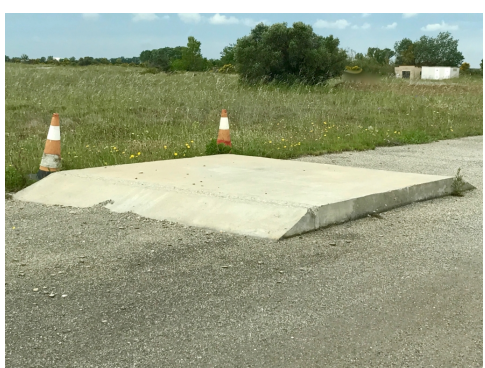

\#3: Speed table

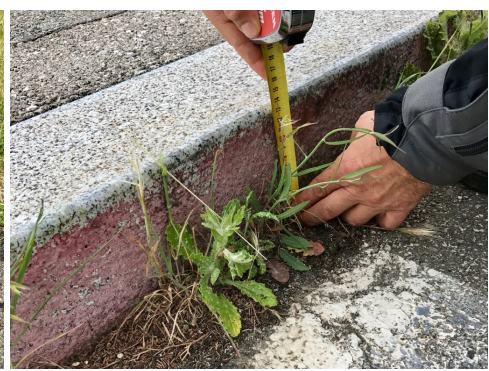

\#6: Kerb

Figure 5 - Road obstacles.

ISO classes. The treatment of short road segments is one main feature of the presented method, since it calculates the PSD for every $\Delta t_{\text {buf }}=1 \mathrm{~s}$. The segment lengths are accumulated simultaneously depending on the road class. This results in a distribution of distances per ISO class for a given road which enables a more detailed classification result for the individual test tracks. Examples are given in Section 6.

\subsection{Road obstacles for impact detection}

For the development and validation of the impact detection strategy, several road obstacles were ridden over with different velocities. The onboard signals were logged for posterior development and validation of the algorithms. Figure 5 illustrates the road obstacles. At first, two railway crossings were tested. Railway crossings are common in rural areas. Depending on the velocity, the discontinuous road surface induces an impact. Next, a relatively large speed table, which can be found in south Europe, south America, and Asia, was tested. The speed table has a short slope up to a height of $h=20 \mathrm{~cm}$ on both sides. This induces an impact at the beginning and at the end of the speed table. Two different sizes of potholes were ridden over to evaluate small road irregularities $(\# 4)$ and larger road defects $(\# 5)$. Both potholes had a depth of $d=5 \mathrm{~cm}$, while the small potholes had a maximum length of $l=30 \mathrm{~cm}$ and the large pothole had a length of $l=1 \mathrm{~m}$. Finally, 
Table 2 - Properties of measured special events.

\begin{tabular}{clcccc}
\hline $\begin{array}{c}\text { Obstacle } \\
\text { No. }\end{array}$ & Description & Special events & $\begin{array}{c}\text { Velocity } \\
\left(\mathrm{m} \mathrm{s}^{-1}\right)\end{array}$ & Mild & Severe \\
\hline$\# 1$ & Railway crossing lateral & 6 & $13.9-16.6$ & 6 & 0 \\
$\# 2$ & Railway crossing angular & 6 & $13.9-16.6$ & 6 & 0 \\
$\# 3$ & Speed table & 6 & $3.1-7.2$ & 6 & 0 \\
$\# 4$ & Pothole small & 15 & $5.5-8.3$ & 15 & 0 \\
$\# 5$ & Pothole large & 12 & $4.7-17.5$ & 12 & 0 \\
$\# 6$ & Kerb & 7 & $3.3-7.2$ & 1 & 6 \\
\hline$\sum$ & & 52 & & 46 & 6 \\
\hline
\end{tabular}

a kerb with a height of $h=14 \mathrm{~cm}$, was tested to demonstrate the threshold between special events and misuse events. The motorcycle was ridden at right angles to the kerb to avoid an accident. Table 2 shows the properties of the test manoeuvres in detail. The number of experiments and the ridden velocity range are illustrated.

Since the set-up allowed no evaluation of the strength of the impact, the test rider was asked to allocate the special events to two groups, mild or severe. A mild special event was defined as an impact that was perceived by the rider, but did not lead to a loss of control or a high feeling of discomfort. A severe special event was defined as the perception of a high degree of discomfort, in combination with a near miss. It was defined as the maximum possible special event, with a transition to misuse events. In total, 52 special events were measured; 46 of them were labelled as mild special events, whereas 6 were labelled as severe special events. Please note that only the kerb manoeuvres were labelled as severe special events.

\section{Road roughness classification}

The present research is based on the theoretical studies about road roughness classification by Gorges et al. [1], to reveal the vehicle-independent distribution of driven road classes. In the previous research, the proposed method of road roughness classification was validated with a full-vehicle model and a numerical simulation. Since the study showed quite promising results, a measurement campaign was carried out and the method has now been tested and validated with a real motorcycle ridden on real test tracks. The main concept of the underlying method will be explained briefly, and the extensions for real operating conditions will then be presented. 


\subsection{Methodology}

The modular road profile classification system utilises the vehicle's transfer functions to compute the excitation from the resultant responses. The velocity-dependant transfer functions had already been calculated from a numerical, full-vehicle model of the motorcycle. The excitation is the longitudinal road profile and the resultant responses are measured by the vehicle's onboard sensors. In the present research, the suspension travel of the front and rear suspension system have been applied as the response signals. The response signals are recorded in the time domain with the help of a sliding window that has a time span of $\Delta t_{\text {buf }}=1 \mathrm{~s}$. Subsequently, the signal extracts are transformed into the time frequency domain with the help of the Fourier transformation. The PSD of the response signal is also computed, which results in $\operatorname{PSD}_{\text {Res }}(f)$. Meanwhile, the transfer functions $H(f)$ are interpolated according to the current velocity $v$. Finally, the PSD of the road profile $\operatorname{PSD}_{\text {Road }}(n)$ can be computed according to Equation (1).

$$
\operatorname{PSD}_{\text {Road }}(n)=\frac{v \operatorname{PSD}_{\text {Res }}(f)}{|H(f)|^{2}} .
$$

After the PSD for a given time or distance segment has been computed, the novel smoothing algorithm, introduced by Gorges et al. [1], is applied. The PSD is smoothed in 10 octave bands, which are proposed by ISO 8608 [2]. The centre frequency in each octave band is calculated by $n_{\mathrm{c}}=2^{\mathrm{EXP}}$. The weighted average values are computed for each octave band using a normalised Gaussian window function. This ensures a good approximation of the PSD at the respective centre frequency, even if only a few values are available within each octave band. This is especially necessary for the evaluation of short test tracks and for the implementation of a real-time road roughness classification. In addition, the PSDs from both the front and the rear suspension sensor can be treated together to achieve a more robust classification result, since more information is evaluated. The result is the smoothed PSD at the ten octave centre frequencies. Subsequently, a minimum distance classifier is applied, which computes the class distance from the ISO [2] classes in every octave band. The class with the lowest error is chosen to be the classification result, as can be found in Gorges et al. [1].

\subsection{Extension for experimental validation}

In the present publication, the validation of the road roughness classification was achieved by riding a real motorcycle on the test tracks. Therefore, the numerical full-vehicle model providing the transfer functions, must describe the real vehicle as well as possible. 


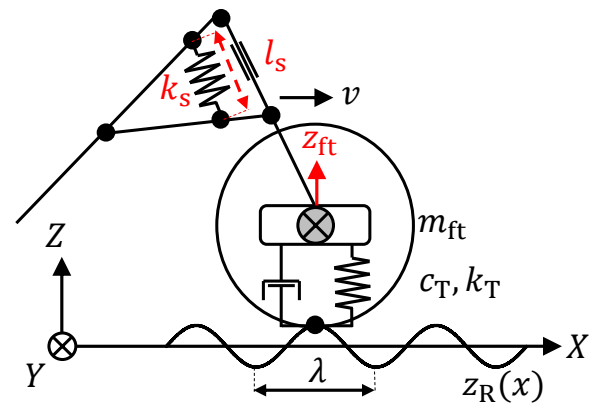

(a) BMW-Telelever suspension system

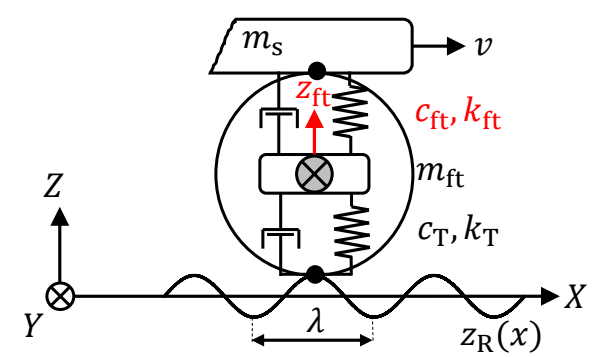

(b) Front part of reduced full-vehicle model [1]

Figure 6 - Derivation of reduced stiffness and damping coefficients.

Since the transfer functions can only be derived from a linear time-invariant model, the nonlinear behaviour of the spring-damper systems must be linearised. In addition, the reduced stiffness and damping coefficients need to be derived, as described by Cossalter [67] and Tanelli et al. [68]. The test motorcycle is designed with a BMW-Telelever front suspension system. It is characterised by one single suspension strut in the $X$ - $Z$-plane. This constructions allows a separation of the suspension functions. The telescopic sliders contribute to the wheel control, whereas the single suspension strut is responsible for the vehicle's comfort and road-holding. In addition, the system is known for its antidive behaviour in braking situations. Figure 6 illustrates the derivation of the reduced stiffness and damping coefficients $k_{\mathrm{ft}}$ and $c_{\mathrm{ft}}$ from the BMW-Telelever front suspension system. The velocity ratio $\beta$ is defined as the ratio between the suspension travel $l_{\mathrm{s}}$ and the vertical wheel movement $z_{\mathrm{ft}}$, see Equation (2).

$$
\beta=\frac{\partial l_{\mathrm{s}}}{\partial z_{\mathrm{ft}}}
$$

The velocity ratio $\beta$ was determined with the help of a multi-body simulation and, for the test motorcycle, is a constant value. For the derivation of the reduced stiffness and damping coefficients $k_{\mathrm{ft}}$ and $c_{\mathrm{ft}}$, the power balance between the wheel force $F_{z \mid \mathrm{ft}}$ and the spring force $F_{\mathrm{S}}$ is applied, see Equation (3).

$$
F_{z \mid \mathrm{ft}} \dot{z}_{\mathrm{ft}}=F_{\mathrm{s}} \dot{l}_{\mathrm{s}}=F_{\mathrm{s}} \frac{\partial l_{\mathrm{s}}}{\partial z_{\mathrm{ft}}} \dot{z}_{\mathrm{ft}} \quad \text { with } \quad F_{\mathrm{s}}=F_{0}+k_{\mathrm{s}}\left(l_{0}-l_{\mathrm{s}}\right)
$$

The spring force $F_{\mathrm{S}}$ is calculated with the spring coefficient $k_{\mathrm{S}}$ and the variation in 
suspension travel $l_{\mathrm{s}}$, where $F_{0}$ is the spring force at the initial suspension travel $l_{0}$. The power balance reduces to

$$
F_{z \mid \mathrm{ft}}=F_{\mathrm{s}} \frac{\partial l_{\mathrm{s}}}{\partial z_{\mathrm{ft}}}=F_{\mathrm{s}} \beta
$$

The reduced stiffness coefficient $k_{\mathrm{ft}}$ is defined as

$$
k_{\mathrm{ft}}=\frac{\partial F_{z \mid \mathrm{ft}}}{\partial z_{\mathrm{ft}}} .
$$

Under the assumption of a constant velocity ratio $\beta$ it reduces to

$$
k_{\mathrm{ft}}=k_{\mathrm{s}} \beta^{2} .
$$

The same approach is used for the derivation of the reduced damping coefficient $c_{\mathrm{ft}}$, and for the derivation of the coefficients for the rear suspension system. In a nutshell, the reduced stiffness and damping coefficients can be derived with the help of the constant velocity ratio $\beta$ and the linearised spring and damper coefficients. In order to complete the derivation of the full-vehicle model, the real motorcycle must be reduced to the sprung mass $m_{\mathrm{s}}$, the front unsprung mass $m_{\mathrm{ft}}$, and the rear unsprung mass $m_{\mathrm{rr}}$.

In addition, to work under real operating conditions, the road roughness classification algorithm also needed some adjustments. First, the front and rear suspension travel response signals are transformed to the vertical wheel movement. This can be achieved with the help of the velocity ratio $\beta$. Moreover, as a preparation for the Fourier transformation, the signal extracts are detrended and windowed. The window function was set empirically to a Tukey window with a taper coefficient $r=0.05$. In addition to the signal preparation for the classification algorithm, the model assumptions must be challenged. In the theoretical study by Gorges et al. [1], the degrees of freedom of the numerical full-vehicle model are the same as for the model from which the transfer functions are derived. Eventually, this led to quite good results. The real motorcycle has roughly the same degrees of freedom in the $X-Z$-plane as the numerical model, but not only the road profile but also the longitudinal dynamics can excite the sprung mass. In the case of significant longitudinal vehicle dynamics, such as accelerating and braking, the sprung mass movement would lead to a false interpretation of the road roughness. Therefore, an on/off logic was implemented to restrict the pitch influence on the road roughness classification. Since no pitch signal was available on the test motorcycle, the longitudinal acceleration was utilised to detect acceleration and brake events. Thus, the classification algorithm pauses when a certain longitudinal acceleration value is exceeded. 


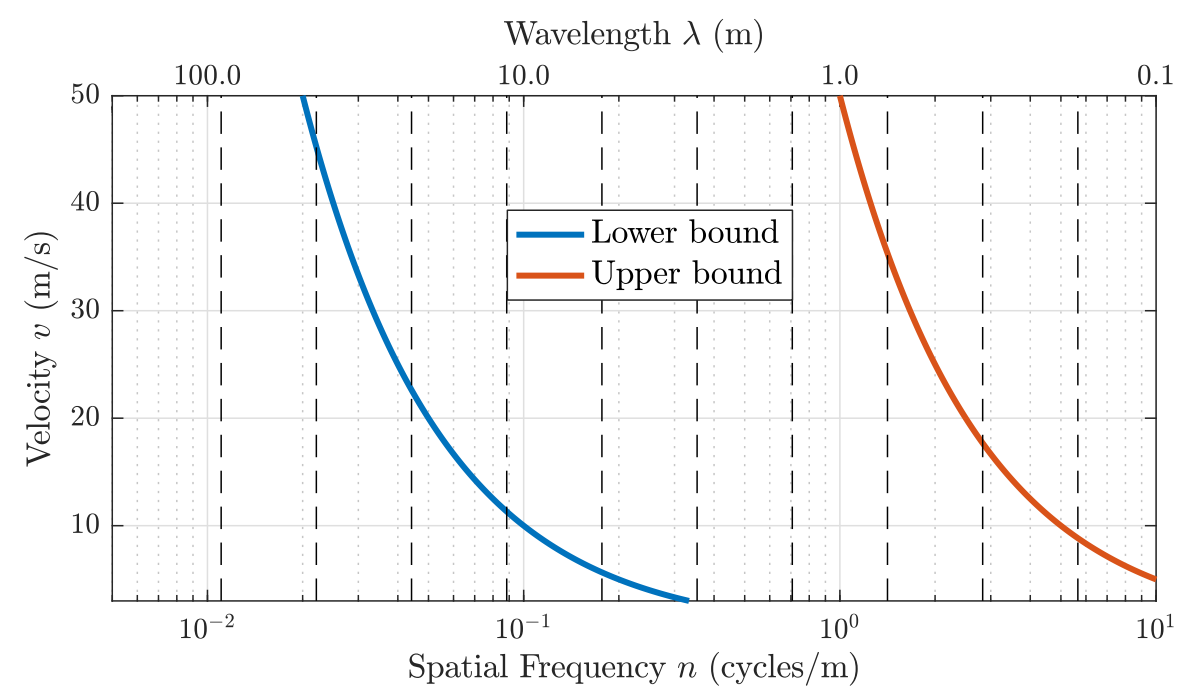

Figure 7 - Lower and upper bound of spatial frequency $n$ depending on the velocity $v$.

Furthermore, the model assumptions are only valid for in-plane dynamics. The algorithm also pauses when the measured angular yaw rate exceeds a certain value, for example during a cornering manoeuvre. Since the classification algorithm is based on the vehicle response, a minimum velocity of $v \geq 3 \mathrm{~m} \mathrm{~s}^{-1}$ must be exceeded so that the roughness classification achieves correct results. When at least one condition is violated, the classification algorithm discards the current signal extract. In this case, the rejected signal extract is classified as unknown roughness.

The sampling rate of the vehicle's bus system is $t_{\mathrm{s}}=0.01 \mathrm{~s}$ and the sliding window for the signal extracts has a time span of $\Delta t_{\text {buf }}=1 \mathrm{~s}$. This combination results in a minimum time frequency of $f_{\min }=1 \mathrm{~Hz}$ and a maximum time frequency of $f_{\max }=50 \mathrm{~Hz}$ after the Fourier transformation. The transformation of the PSD from the time frequency $f$ to the spatial frequency $n$, depends on the velocity $v$. As a consequence, the PSD is defined by a restricted spatial frequency domain depending on the velocity $v$. Figure 7 illustrates the lower and upper bounds of the restricted spatial frequency domain of the transformed PSD as functions of the velocity. Because there is a moving spatial frequency domain, the classification algorithm was designed to handle varying spatial frequencies. It can be seen that independent of the velocity $v$, a minimum of five octave bands can be evaluated, which are highlighted with the dashed lines. 


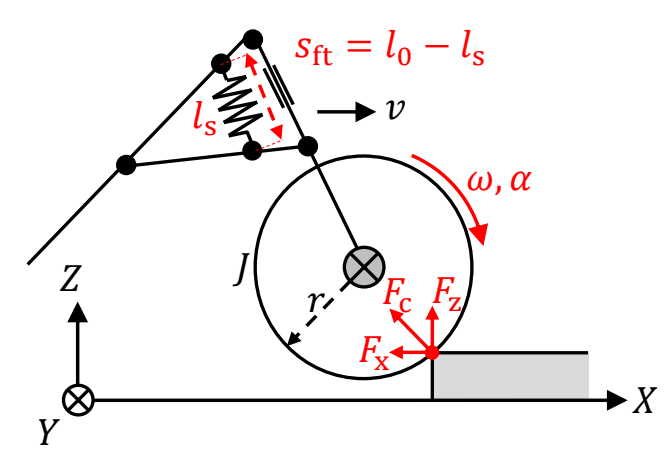

Figure 8 - Schematic view of an impact.

\section{Impact detection}

The road roughness classification system is designed to classify the road roughness independently of the velocity. However, from the viewpoint of durability, the combination of the vehicle velocity and the road surface roughness induces varying loads that need further investigation. Therefore, an impact detection strategy was developed to detect and classify special events, for example passing over a kerb.

\subsection{Model of an impact event}

Figure 8 shows a schematic view of an impact. The angular front wheel velocity $\omega$ and the variation in suspension travel $s_{\mathrm{ft}}$ are available for the detection of special events. The variation in suspension travel is given by $s_{\mathrm{ft}}=l_{0}-l_{\mathrm{s}}$, where $l_{0}$ is the length of the uncompressed spring. The impact induces a contact force $F_{c}$, which is decomposed into its components $F_{x}$ and $F_{z}$. The contact force induces a change in the angular momentum $L$ of the front wheel, see Equation (7).

$$
\Delta L=J \alpha, \quad \alpha=\frac{\mathrm{d} w}{\mathrm{~d} t}, \quad \omega=\frac{v}{r} .
$$

\subsection{Signal processing and feature extraction}

Figure 9 shows the onboard signals for the angular front wheel velocity $\omega$ and the variation in suspension travel $s_{\mathrm{ft}}$ during a kerb crossing, together with their time derivatives, angular wheel acceleration $\alpha$ and suspension travel velocity $\dot{s}_{\mathrm{ft}}$. The change in the angular momentum can be seen as a peak in the angular wheel velocity $\omega$, or in the angular acceleration $\alpha$ of the front wheel. In addition, the $z$-component of the contact force lifts 

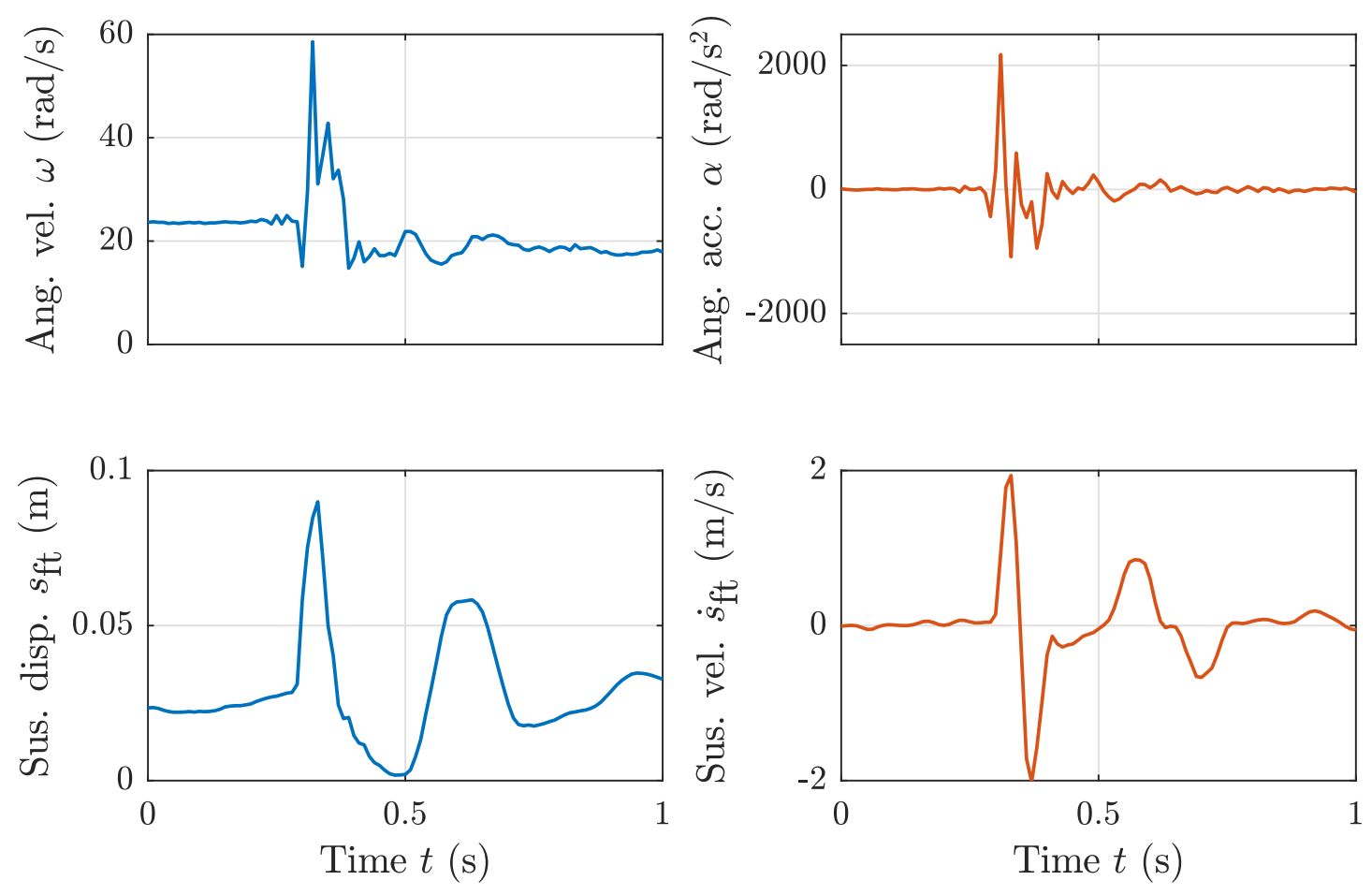

Figure 9 - Onboard signals during kerb crossing.

the wheel over the kerb and compresses the suspension spring. Hence, the impact can also be seen in the suspension signal $s_{\mathrm{ft}}$ and its derivative, the suspension travel velocity $\dot{s}_{\mathrm{ft}}$. The kerb crossing manoeuvre is representative for all the other special events, because all the events have in common that the impact induces an $x$ - and $z$-component of the contact force. For the application of a classification method, feature extraction from the input signals is necessary. The authors tested some of the statistical key numbers, for example standard deviation, variance, root mean squared error, and peak-to-peak on sliding windows with a time span of $\Delta t_{\text {buf }}=1 \mathrm{~s}$. Studies have shown that these key numbers are highly correlated and in terms of feature selection, the authors decided to pick the peak-to-peak values of the angular wheel acceleration $\alpha$ and the suspension velocity $\dot{s}_{\mathrm{ft}}$ as features.

The choice of the time derivatives of the original measured onboard signals angular velocity $\omega$ and variation in suspension travel $s_{\mathrm{ft}}$, has two major benefits: First, the time derivatives act as a numerical high-pass filter. Thus, the influence of sensor drifts and offsets is prevented. Second, particularly for the suspension travel signal, only the time derivative distinguishes between a spring compression as a result of a special event, and a spring compression as a result of vehicle dynamics, for example, braking and acceleration 


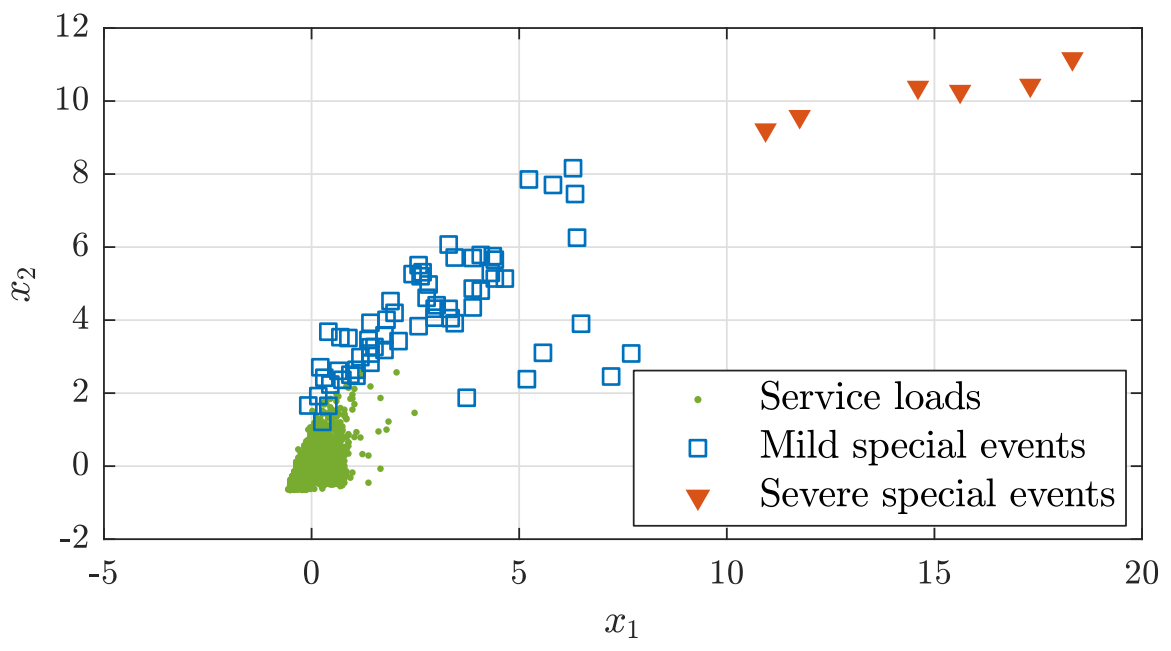

Figure 10 - Scatter plot of the training set.

events. As a consequence, the suspension travel velocity $\dot{s}_{\mathrm{ft}}$ is more practical for the robust detection of special events. Since the units and the dimensions of the selected features are different, it is common in machine learning techniques to standardise the input variables. Accordingly, the features have been standardised by the z-score, and are henceforth referred as $x_{1}$ and $x_{2}$ as given in Equation (8).

$$
x_{1}=\frac{\left(\alpha-\mu_{\alpha}\right)}{\sigma_{\alpha}} \quad \text { and } \quad x_{2}=\frac{\left(\dot{s}_{\mathrm{ft}}-\mu_{\dot{s}_{\mathrm{ft}}}\right)}{\sigma_{\dot{s}_{\mathrm{ft}}}} .
$$

\subsection{Training set}

Figure 10 shows a scatter plot of the observations in the $x_{1}-x_{2}$ plane. For this, the measured onboard signals from the 52 special events were processed using the sliding window, feature extraction, and standardisation. For the classification and detection of special events, it is necessary to also include observations that represent the normal usage of the motorcycle. For this reason, the measurements for test tracks No. 1-6 from the road roughness classification, were also processed and are labelled as service loads. In addition, several measurements for dynamic manoeuvres have been provided, so that the classifier can distinguish between normal usage and special events. For a robust classification algorithm, it is essential to provide as many observations as possible in the training set. On the one hand, the scatter plot shows a clear distinction between mild and severe special events, which confirms the correct labelling of the observations. On the other hand, the transition between service loads and mild special events is ambiguous. Some normal usage manoeuvres also lead to a spring compression and/or an angular 
wheel acceleration, for example intense braking events. In total, the training set consists of 2459 service loads, 46 mild special events, and 6 severe special events.

\section{Results and Discussion}

This section contains the evaluation of the methods presented. The results of the road roughness classification algorithm were compared to the classification results of the laserscanned road profiles. For the impact detection strategy, different machine learning techniques were evaluated and validated with the k-fold cross-validation method.

\subsection{Validation of the road roughness classification}

Since the road roughness classification algorithm determines a road class for every $\Delta t_{\text {buf }}=$ $1 \mathrm{~s}$, the resultant distances per road class are accumulated. As discussed in Section 3.2, a single classification for the complete measured road profile leads to inaccurate results. Therefore, the measured road profile was segmented according to the distance for which the motorcycle stayed within the sliding window. Only this method enables a comparison of the results. For example, at test track No. 1, the motorcycle had a mean velocity of $v_{\text {mean }}=26 \mathrm{~m} \mathrm{~s}^{-1}$ and therefore the segment length was set at $l_{\text {seg }}=26 \mathrm{~m}$. On the one hand, this method guarantees that the same number of classification results are compared. On the other hand, it ensures that the measured road profile is evaluated at the same spatial frequencies as the classification algorithm that uses the response signals. Figure 11 shows the validation results for test tracks No. 1-6, while CRG data represents the measured road profile. The percentage of correctly classified segments is shown in Table 3.

Table 3 - Results of the road roughness classification.

\begin{tabular}{lcccccc}
\hline Test track No. & $\# 1$ & $\# 2$ & $\# 3$ & $\# 4$ & $\# 5$ & $\# 6$ \\
\hline Correct classified (\%) & 99.8 & 87.5 & 88.2 & 91.8 & 95.7 & 95.4 \\
\hline
\end{tabular}

Test track No. 1 is characterised by the smoothest surface, and was almost completely classified as an ISO class A road by both the classification algorithm and the measured data. A classification result of $99.8 \%$ was achieved. The main classification result for test track No. 2 is ISO class B. However, a third of the test track was classified to ISO class A and C. A coincidence of $87.5 \%$ was achieved. A similar result shows the comparison of test track No. 3, where a coincidence of $88.2 \%$ was obtained. Almost $80 \%$ of test track No. 4 was classified as ISO class B and roughly $20 \%$ as ISO class C. A classification 


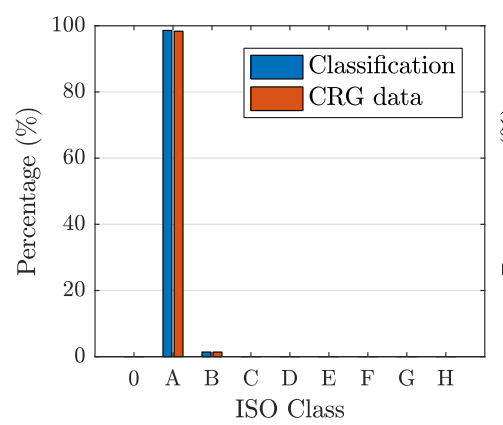

\#1: High-speed test track

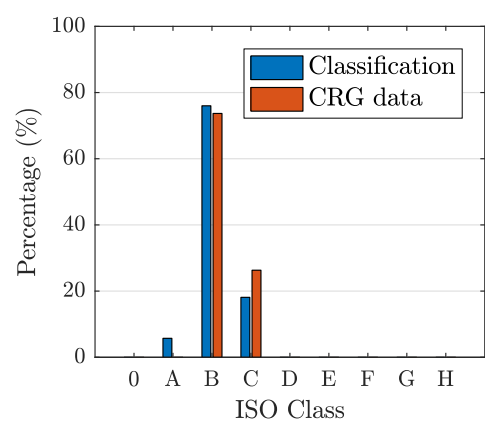

\#4: Fine cobblestone

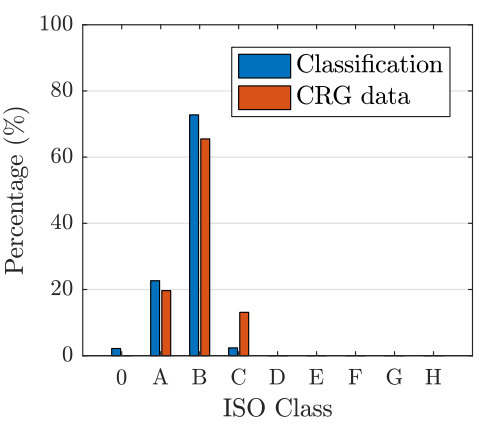

\#2: Bumpy country road

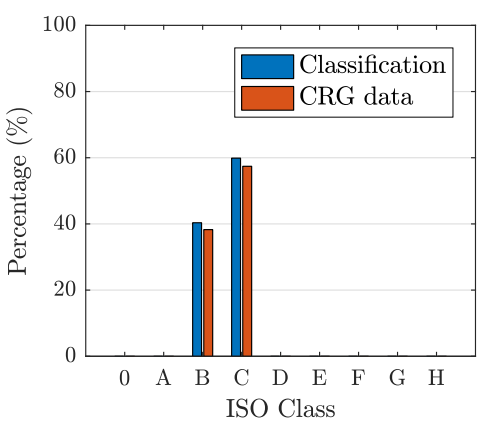

\#5: Dilapidated concrete panels

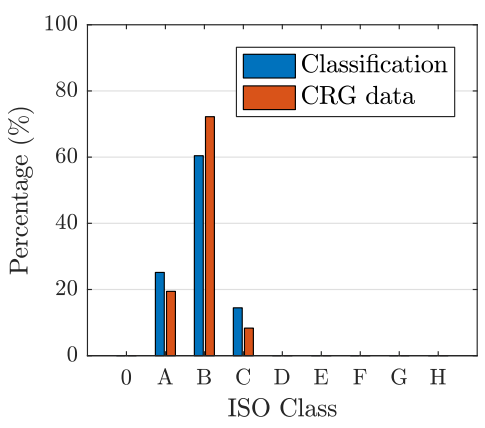

\#3: Dilapidated country road

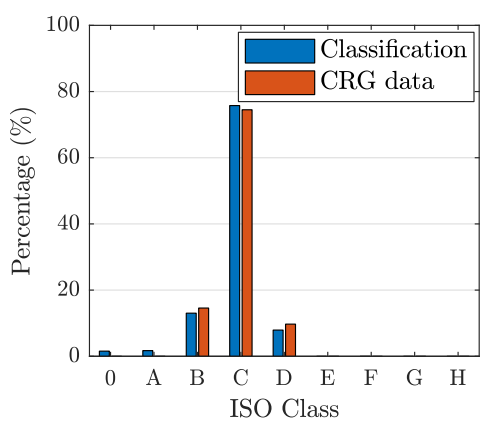

\#6: Coarse cobblestone

Figure 11 - Results of the road roughness classification for test tracks No. 1-6.

result of $91.8 \%$ was achieved. In contrast, test track No. 5 was mainly classified as ISO class $\mathrm{C}(\approx 60 \%)$. A smaller part was classified as ISO class B $(\approx 40 \%)$. An overall classification result of $95.7 \%$ was obtained for this test rack. The roughest test track in the present measurement campaign is test track No. 6, which was classified as almost $80 \%$ ISO class C, and some parts as class B and D. An overall classification result of $95.4 \%$ was achieved.

Figure 12 shows the validation results for the test tracks No. 7-9. Due to their loose surfaces, these test tracks have no measurement data available, as discussed in Section 3.2. Test track No. 7 was classified as a mixture of road classes, from ISO class A to C. Test track No. 8 was subjectively perceived as being much rougher and harsher than test track No. 7, as the relative comparison of the classification results also shows. This test track was mainly classified as ISO class C-E. The highest classification results were obtained at test track No. 9, which was mainly classified as ISO class D-E. On the one hand, the classification results obtained reflect the subjective feeling of roughness quite well in comparison to the roads that have been validated with measurements. On the other hand, the relative comparison of the classification results of the test tracks No. 7-9, represent the rider's perception of increasing roughness. In summary, the road 


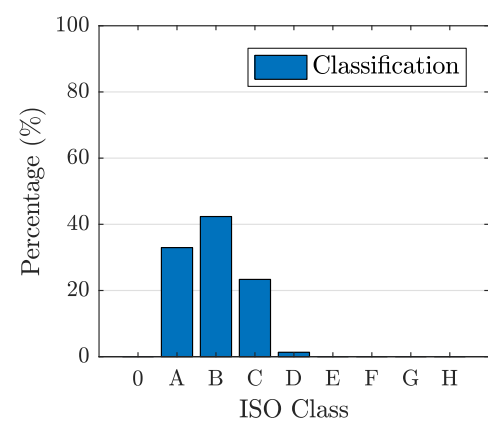

\#7: Gravel road

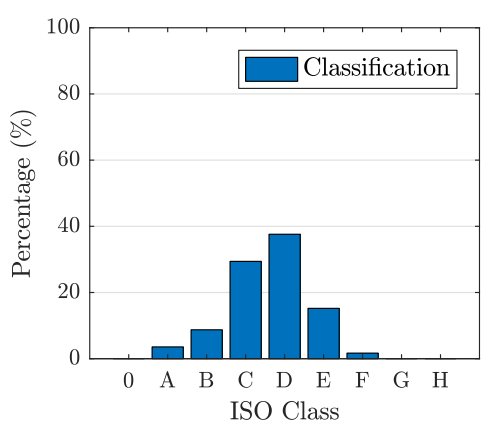

\#8: Rough unpaved roads

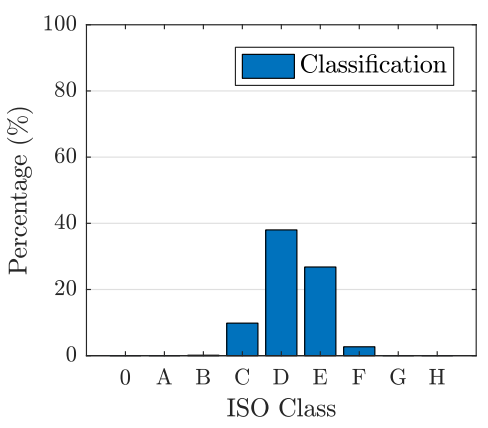

\#9: Enduro fun park

Figure 12 - Results of the road roughness classification for test tracks No. 7-9.

roughness classification algorithm developed, achieves classification results of more than $87 \%$, which is sufficient for the purpose of road classification in terms of customer usage profiles. In contrast to the simplified classification method discussed in Section 3.2, the method presented of dividing road segments, leads to a more detailed classification result. Compared to Table 1, the classification result is more detailed. This was also expected due to the non-linear shape of the PSDs of the measured road profiles, compare Figure 4. Accordingly, a single road can be characterised by different road classes at different spatial frequencies. For this reason, the method presented of dividing road segments, is proposed to be preferable for classifying roads and test tracks. The results from the divided road segments need to be summarised to achieve a distribution of driven distances per road class.

The differences between the road roughness classification results and the measured road profiles, have two reasons: First, the full-vehicle model is a simplification of the real motorcycle. This implies that nonlinearities are ignored, and reduced stiffness and damping coefficients have been applied. Moreover, in the event of small height differences, for example, gravel, the tyre smooths the road profile. On the contrary, small gaps cannot be measured correctly due to the geometric dimensions of the tyre. Furthermore, the model does not consider the influence of longitudinal dynamics on the response signals, as discussed in Section 4. Second, the method of validation has some potential error sources. The segmentation of the measured and the driven road is not equally accurate. This means that the start and end points of the test tracks have been determined by the GPS position of both the measurement vehicle and the test motorcycle. This might be a source of error in the evaluation of the individual road segments. In addition, the trajectory of the motorcycle and especially the lateral position on the test track, is ambiguous and therefore, the correct longitudinal road profile cannot be derived from the 
CRG-data. During the measurement campaign, the rider attempted to ride in the centre of the lane as much as possible. Hence, the longitudinal profile of the three-dimensional measurement data has also been derived from the middle of the lane. In summary, model and systematic failures affect the validation of the road roughness classification.

\subsection{Validation of the impact detection}

After the training set had been created, different machine learning techniques were applied. In the terminology of machine learning, the current problem is described as supervised machine learning. According to Suthaharan [69], in supervised learning, all classes are known and class boundaries are well defined in the given training set. The objective is to generate rules and to train a classifier, using the training set, so that new, unlabelled data can be classified. This is also called classification. Although this classification problem is trivial, it is well suited for a study on the principles of classification algorithms. Especially because the feature space can be plotted because the problem is two-dimensional. Hence, nine popular classification methods were tested on the training set, as can be seen in Table 4. The accuracy of the classifiers was tested by the k-fold cross-validation, where the number of subsets was set to $k=5$. In machine learning, the problem with validation is the division of the data set into a training set and a testing set. There is a target conflict between the maximum amount of information in the training set to provide the best possible training data, and the maximum of testing data to evaluate the performance of the trained classifier. Cross-validation partitions the data set into $k$ folds, and for each fold, a model is trained using the out-of-fold observations. The performance is tested using the in-fold data. This procedure is repeated $k$ times, and finally, the average performance over all folds is calculated. This method has the advantage that all observations are used for training and testing. Cross-validation is only used for the evaluation of the classifier, in terms of accuracy. The final classifier was trained using the complete training set. The classifiers are ordered depending on the normalised prediction speed based on the fastest algorithm. Hence, the fastest classifier is the logistic regression.

Overfitting is a phenomenon in which the classifier models the training data too well, so that the performance on new data decreases. In the present study, the problem of overfitting is handled by the so-called decision surfaces, where the solution space is evaluated by the expectation and experience of the user. Hence, the benchmark shape was plotted in the form of decision surfaces, as illustrated in Figure 13. According to the authors' expectation, a threshold on both features $x_{1}$ and $x_{2}$ must be exceeded to distinguish between service loads and special events. In addition, severe special events 
Table 4 - Properties of classification methods.

\begin{tabular}{clccc}
\hline No. & Classifier & Prediction speed & Overfitting & Accuracy \\
\hline$\# 1$ & Logistic Regression & 1 & $\mathrm{n}$ & 99.60 \\
$\# 2$ & Decision Tree & 1.5 & $\mathrm{n}$ & 99.64 \\
$\# 3$ & Discriminant Analysis (linear) & 3.5 & $\mathrm{n}$ & 99.57 \\
$\# 4$ & Discriminant Analysis (quadratic) & 4.5 & $\mathrm{y}$ & 98.93 \\
$\# 5$ & Naive Bayes & 4.5 & $\mathrm{n}$ & 99.01 \\
$\# 6$ & SVM (linear) & 5 & $\mathrm{n}$ & 99.41 \\
$\# 7$ & KNN, (k=1) & 7 & $\mathrm{n}$ & 99.53 \\
$\# 8$ & SVM (quadratic) & 8 & $\mathrm{n}$ & 99.76 \\
$\# 9$ & SVM (Gaussian) & 15 & & 99.37 \\
\hline
\end{tabular}

are also distinguished from mild special events by the exceeding of thresholds. The boundaries need not necessarily be in linear form, they could also be in radial form. This benchmark shape helps to evaluate the problem of overfitting and the treatment of future data. It is more an orientation for the user than a nominal target. Since all the classifiers achieved an accuracy of almost $100 \%$, further investigations have been carried out. Due to the two-dimensional problem, it is possible to plot the decision surfaces for all the classifiers, see Figure 13. This opportunity offers a benefit, because the decision surfaces show graphically how the classifiers separate the observations. In addition, the treatment of future data can be estimated. First, a multinomial logistic regression was tested, which is fast and easy to implement. The algorithm calculates probabilities for each observation. The linear behaviour of the classifier is readily identifiable. The decision surfaces show a good separation of the classes. Second, a coarse binary decision tree with a maximum number of four leafs, was trained. Decision trees are easy to interpret and make fast predictions. The boundaries of the decision surfaces are parallel to the coordinate system, which is characteristic for decision trees. This classifier comes closest to the benchmark shape.

Third, a linear and a quadratic discriminant analysis were tested. The algorithms assume that different classes generate data based on different Gaussian distributions. Although these algorithms make fast predictions, the decision surfaces are not satisfactory. For the linear discriminant analysis, the boundary of severe special events is too close to the mild special events. Overfitting occurs for the quadratic discriminant analysis, which means that the classifier is trained specifically for the training set, but unknown data could be incorrectly classified. Next, a naive Bayes classifier was tested, which is based on the Bayes' theorem. It assumes independence between features. In the present study, the features are highly correlated, but nevertheless the classifier shows a good performance. The shape of the decision surfaces are satisfactory. Subsequently, the family of support vector machines (SVM) was evaluated with different kernel functions. 




\#0: Benchmark shape

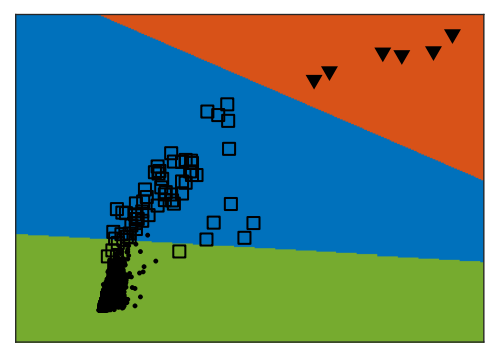

\#1: Logistic Regression

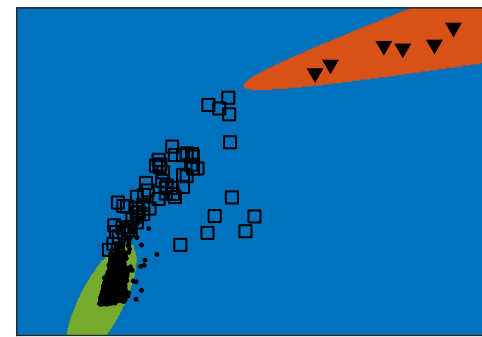

\#4: Quadratic Discriminant

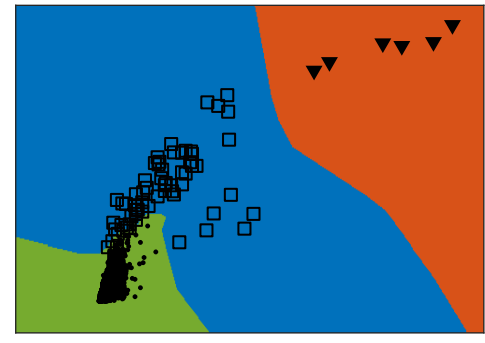

$\# 7: \mathrm{KNN},(k=1)$

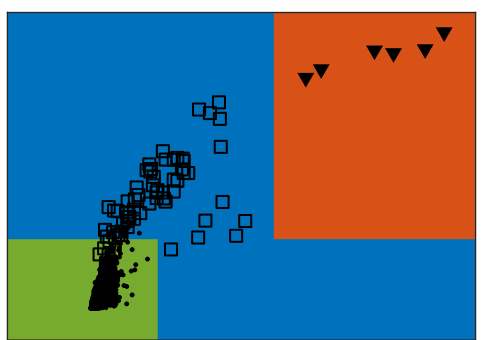

\#2: Decision Tree

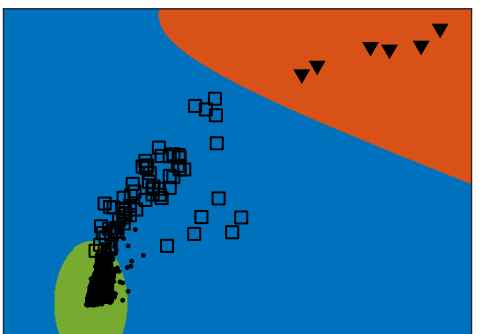

\#5: Naive Bayes

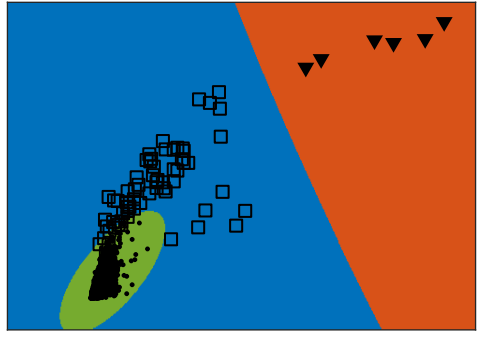

\#8: SVM (quadratic)

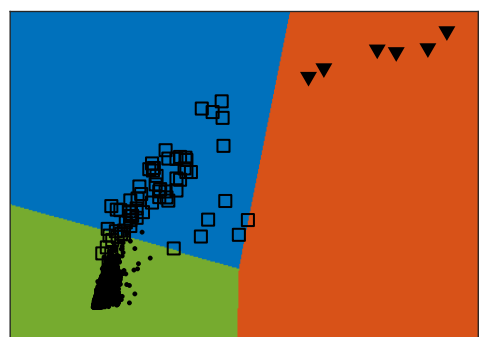

\#3: Linear Discriminant

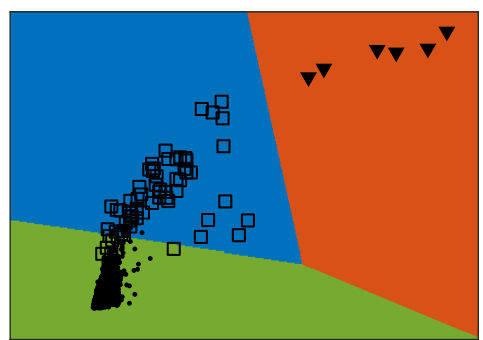

\#6: SVM (linear)

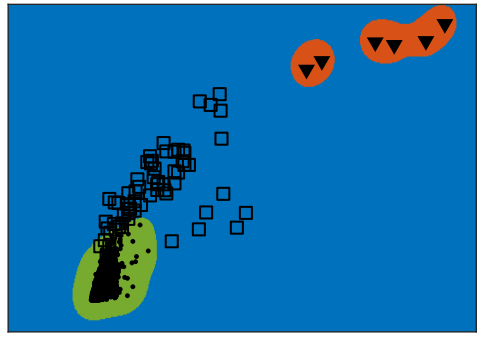

\#9: SVM (Gaussian)

Figure 13 - Decision surfaces for different classifiers.

SVMs classify data by finding the hyperplane that separates the data with the largest margin between the classes. The linear implementation shows a good separation between the classes, while the space for special events is too large at the quadratic implementation. The implementation with the Gaussian kernel function is characterised by strong overfitting, which can be seen on the small islands to detect severe special events. How- 
Table 5 - Confusion matrix of binary decision tree.

\begin{tabular}{|c|c|c|c|c|c|}
\hline & & Service loads & $\begin{array}{l}\text { Actual Class } \\
\text { Mild special events }\end{array}$ & Severe special events & TPR (\%) \\
\hline \multirow{3}{*}{ 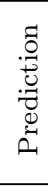 } & Service loads & 2456 & 6 & 0 & 99.8 \\
\hline & Mild special events & 3 & 40 & 0 & 93.0 \\
\hline & Severe special events & 0 & 0 & 6 & 100.0 \\
\hline & PPV (\%) & 99.9 & 87.0 & 100 & 99.6 \\
\hline
\end{tabular}

ever, SVMs show a medium to slow prediction performance, especially for multi-class problems. Finally, the $k$-Nearest-Neighbour classifier was evaluated with $k=1$. This algorithm tries to classify observations depending on the $k$ closest points. This technique has a medium prediction speed compared to the others. Kotsiantis et al. [70] provide a review of classification methods.

The authors decided to implement the binary decision tree for the prediction of future observations. The classifier shows a high accuracy after k-fold cross-validation and the decision surfaces clearly separate the classes. In addition, the classifier represents the benchmark shape and is fast on the prediction of future data. After the 5-fold crossvalidation, the algorithm shows an accuracy of $99.6 \%$, as can be seen in Table 5 . The numbers within table represent the number of observations. The last column shows the True Positive Rate (TPR) and the last row shows the Positive Predictive Value (PPV). For example, $87 \%$ of the actual mild special events have been predicted correctly and $93 \%$ of the predicted mild special events are actually true. It is important, that severe special events are predicted and classified $100 \%$ correctly, since they have the highest impact on the vehicle. The losses between service loads and mild special events are acceptable, since the scatter plot also shows an ambiguous assignment. However, this behaviour was expected. Figure 14 illustrates the final binary decision tree with four leaves.

In summary, a sliding window buffers the angular front wheel velocity $\omega$ and the variation in suspension travel $s_{\mathrm{ft}}$ for a time span of $\Delta t_{\mathrm{buf}}=1 \mathrm{~s}$. Subsequently, the time derivatives of these two signals are calculated. The peak-to-peak values are extracted and normalised by the z-score, to provide the input variables $x_{1}$ and $x_{2}$. The predictor is a trained classifier of decision tree type, and continuously classifies the observations into service loads, mild special events, and severe special events.

The resultant classifier is based on the training set achieved from the test motorcycle riding with different velocities over the road obstacles. The application of the classifier is dedicated to this experimental set-up. Varying velocities are covered by the generation of 


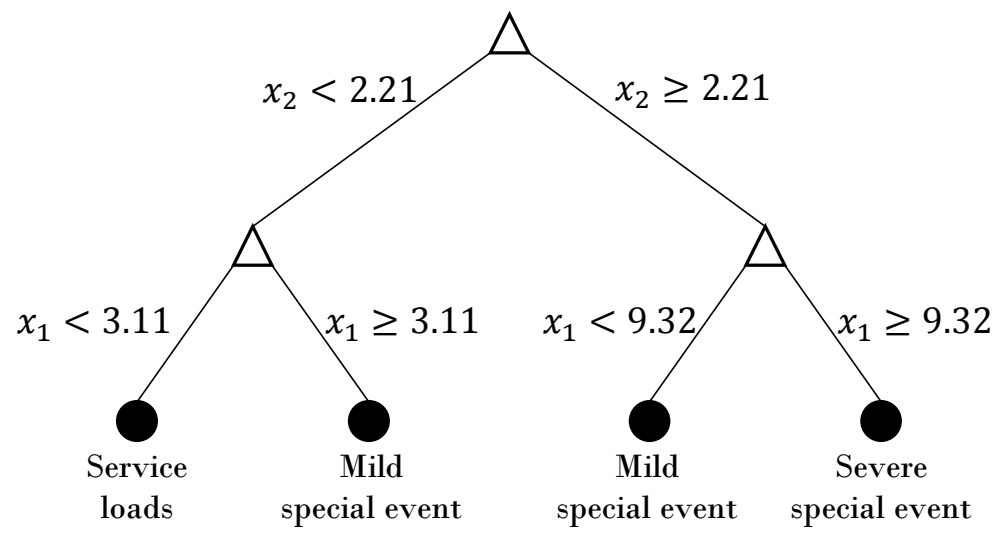

Figure 14 - Binary decision tree.

the training set, whereas a different type of motorcycle would probably lead to incorrect classification results. Thus, it is recommended to generate individual training sets for other types of motorcycles. Both measuring the front suspension signal as well as riding over the presented obstacles is part of the endurance tests at the product validation. Thus, there is no additional effort except of labelling the special events.

\section{Conclusion}

The objective of this research was the experimental validation of a road roughness classification method and the development of an impact detection strategy, in the context of durability engineering. Both methods were validated with the help of a measurement campaign. Nine test tracks and six obstacles were evaluated, while the test tracks had been surveyed by a laser scanner in a previous BMW study. The methods developed utilise the vehicle's onboard signals and are therefore suitable to collect customer usage profiles in terms of field data.

The road roughness classification algorithm was experimentally validated, after the proof of concept had been achieved theoretically in a previous publication by Gorges et al. [1]. The method was extended to work under real operating conditions. The results show an accuracy of more than $87 \%$ on the test tracks. Furthermore, the validation method shows that the evaluation of divided road segments leads to a more detailed classification result, compared to the classification results achieved by processing the complete road profile. This fact confirms the application of the developed road roughness classification method, because in the presented real-time implementation, the road roughness is also processed in small road segments depending on the velocity. In summary, the road 


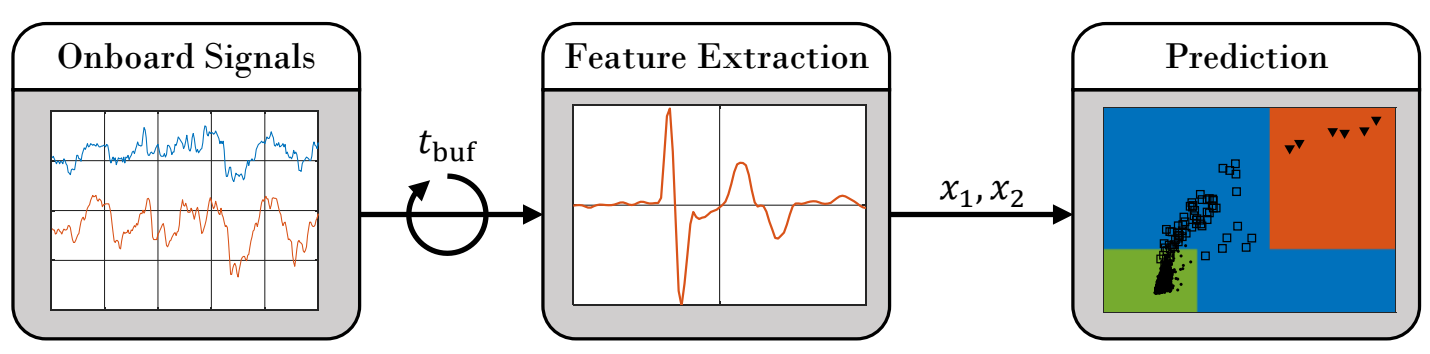

Figure 15 - Flowchart of the impact detection strategy.

roughness classification algorithm was proved to achieve adequate results on real test tracks. The methodology was experimentally validated, even on rough, unpaved roads, and can be implemented into new products to collect customer usage profiles. The presented algorithm requires a linearised full-vehicle model of the motorcycle, the velocity, and at least one suspension deflection signal, as inputs.

The road roughness classification system continuously classifies the road roughness, independently of the velocity and the vehicle. From the viewpoint of durability, only the combination of road roughness and vehicle velocity determines whether the induced loads are service loads, special events, or misuse events. Therefore, the impact detection strategy was developed. Based on the same input signals, velocity and spring deflection, a classifier was trained for detecting special events. Different road obstacles were ridden over with different velocities to generate a broadly distributed data set. The test rider divided the events into two classes: mild special events and severe special events. The peak-to-peak values of the time derivatives of the angular front wheel velocity and the suspension travel, were chosen as features. After normalisation of the features, different machine learning techniques were evaluated on the given training set, in terms of prediction speed, accuracy, overfitting, and shape of the decision surfaces. The decision tree was chosen, with an accuracy of $99.6 \%$. The validation was performed by a k-fold cross-validation. The results show that a distinction between service loads, mild special events, and severe special events is possible. The impact detection strategy can easily be appended to the existing road roughness classification system, since the algorithm works with the same onboard signals and the same sliding window, as the road roughness classification. A schematic flowchart of the impact detection strategy is provided in Figure 15. Further research should investigate the robustness of the classifier against changes of the vehicle attributes; for example, changes of the vehicle weight or manipulations of the suspension and tyre characteristics.

All of the algorithms developed work in real time. Thus, an implementation into exist- 
ing electronic control units is possible. The distribution of driven road classes improves vehicle design targets. Furthermore, it enables a virtual load acquisition and makes the generation of synthetic road profiles possible. In addition, the amount and strength of special events helps to define maximum loads. The combination of distributed road classes and special events, completes the description of customer usage profiles. Further research should investigate the possibilities of a real-time application for the information gathered. For example, the knowledge of severe special events could enable applications for predicted maintenance. Moreover, the statistical treatment of the gathered field data and the derivation of design loads, needs investigation. Finally, the interaction between field data collection and a virtual load acquisition, must be examined from the viewpoint of the vehicle design process.

\section{References}

[1] C. Gorges, K. Öztürk and R. Liebich. 'Road classification for two-wheeled vehicles'. In: Vehicle System Dynamics 56.8 (2018), pp. 1289-1314.

[2] ISO. Mechanical vibration - Road surface profiles - Reporting of measured data. Standard No. 8608:2016. Geneva, Switzerland: ISO, 2016.

[3] C. Matz. 'Online Berechnung von Fahrwerkskräften auf Basis von Onboard-Sensorik'. PhD thesis. Clausthal: TU Clausthal, 2015.

[4] K. Pötter. 'Structural durability and integrity in vehicle design'. In: 18th European Conference on Fracture. Dresden, Germany: DVM, Aug. 2010.

[5] P. Johannesson and M. Speckert, eds. Guide to load analysis for durability in vehicle engineering. Chichester: Wiley, 2013, p. 434.

[6] M. Köhler, S. Jenne, K. Pötter et al. Load Assumption for Fatigue Design of Structures and Components. Berlin, Heidelberg: Springer, 2017, pp. 1-226.

[7] M. Hauke. 'Simulation des Missbrauchverhaltens von Gesamtfahrzeugen'. PhD thesis. Munich, Germany: TU München, 2004.

[8] C. Berger, K.-G. Eulitz, P. Heuler et al. 'Betriebsfestigkeit in Germany - an overview'. In: International Journal of Fatigue 24.6 (June 2002), pp. 603-625.

[9] C. Gorges, K. Öztürk and R. Liebich. 'Customer loads of two-wheeled vehicles'. In: Vehicle System Dynamics 55.12 (2017), pp. 1842-1864.

[10] A. González, E. J. O'brien, Y.-Y. Li et al. 'The use of vehicle acceleration measurements to estimate road roughness'. In: Vehicle System Dynamics 46.6 (2008), pp. 483-499.

[11] N. K. Harris, A. González, E. J. O'Brien et al. 'Characterisation of pavement profile heights using accelerometer readings and a combinatorial optimisation technique'. In: Journal of Sound and Vibration 329.5 (2010), pp. 497-508. 
[12] H. M. Ngwangwa, P. S. Heyns, F. J. J. Labuschagne et al. 'Reconstruction of road defects and road roughness classification using vehicle responses with artificial neural networks simulation'. In: J. Terramechanics 47.2 (2010), pp. 97-111.

[13] M. Doumiati, A. Victorino, A. Charara et al. 'Estimation of road profile for vehicle dynamics motion: experimental validation'. In: American Control Conference. San Francisco: IEEE, June 2011, pp. 5237-5242.

[14] W. Yu, X. Zhang, K. Guo et al. 'Adaptive real-time estimation on road disturbances properties considering load variation via vehicle vertical dynamics'. In: Math. Probl. Eng. 2013 (2013), pp. 1-9.

[15] M. Doumiati, S. Erhart, J. Martinez et al. 'Adaptive control scheme for road profile estimation: application to vehicle dynamics'. In: The International Federation of Automatic Control. Cape Town: IFAC, Aug. 2014, pp. 8445-8450.

[16] M. Doumiati et al. 'Road profile estimation using an adaptive Youla-Kučera parametric observer: Comparison to real profilers'. In: Control Engineering Practice 61.2002 (Apr. 2017), pp. 270-278.

[17] J. C. Tudón-Martínez, S. Fergani, O. Sename et al. 'Online road profile estimation in automotive vehicles'. In: European Control Conference (ECC). Strasbourg: ECC, June 2014, pp. 2370-2375.

[18] J. C. Tudón-Martínez, S. Fergani, O. Sename et al. 'Adaptive road profile estimation in semiactive car suspensions'. In: IEEE Transactions on Control Systems Technology 23.6 (2015), pp. 2293-2305.

[19] Z. Wang et al. 'Road profile estimation for suspension system based on the minimum model error criterion combined with a Kalman filter'. In: J. Vibroengineering 19.6 (Sept. 2017), pp. 4550-4572.

[20] Z. Wang et al. 'Suspension system state estimation using adaptive Kalman filtering based on road classification'. In: Vehicle System Dynamics 55.3 (Mar. 2017), pp. 371-398.

[21] Y. Qin et al. 'Road excitation classification for semi-active suspension system with deep neural networks'. In: J. Intell. Fuzzy Syst. 33.3 (Aug. 2017), pp. 1907-1918.

[22] Y. Qin et al. 'Road profile estimation for semi-active suspension using an adaptive Kalman filter and an adaptive super-twisting observer'. In: $2017 \mathrm{Am}$. Control Conf. Seattle, USA: IEEE, May 2017, pp. 973-978.

[23] Y. Qin et al. 'Road excitation classification for semi-active suspension system based on system response'. In: Journal of Vibration and Control (2017), pp. 1-17.

[24] D. Ben Hassen et al. 'Road profile estimation using the dynamic responses of the full vehicle model'. In: Applied Accoustics May (Dec. 2017), pp. 0-1.

[25] M. Burger. 'Calculating road input data for vehicle simulation'. In: Multibody Syst. Dyn. 31.1 (2013), pp. 93-110. 
[26] W. Fauriat, C. Mattrand, N. Gayton et al. 'Estimation of road profile variability from measured vehicle responses'. In: Vehicle System Dynamics 54.5 (2016), pp. 585-605.

[27] M. Yousefzadeh, S. Azadi and A. Soltani. 'Road profile estimation using neural network algorithm'. In: Journal of Mechanical Science and Technology 24.3 (2010), pp. 743-754.

[28] H. Imine, Y. Delanne and N. K. M'Sirdi. 'Road profile input estimation in vehicle dynamics simulation'. In: Vehicle System Dynamics 44.4 (2006), pp. 285-303.

[29] J. J. Rath, K. C. Veluvolu and M. Defoort. 'Simultaneous estimation of road profile and tire road friction for automotive vehicle'. In: IEEE Transactions on Vehicular Technology 64.10 (2015), pp. 4461-4471.

[30] W. Jeong, K. Yoshida, H. Kobayashi et al. 'State estimation of road surface and vehicle system using a Kalman filter'. In: JSME Int. J. 33.4 (1990), pp. 528-534.

[31] T. P. Nordberg. 'An iterative approach to road/profile identification utilizing wavelet parameterization'. In: Vehicle System Dynamics 42.6 (2004), pp. 413-432.

[32] Y. Qin, M. Dong, F. Zhao et al. 'Road profile classification for vehicle semi-active suspension system based on adaptive neuro-fuzzy inference system'. In: Conference on Decision and Control (CDC). Osaka: IEEE, Dec. 2015, pp. 1533-1538.

[33] Y. Qin, M. Dong, R. Langari et al. 'Adaptive hybrid control of vehicle semiactive suspension based on road profile estimation'. In: Shock Vib. 2015.1 (2015), pp. 113.

[34] A. Solhmirzaei, S. Azadi and R. Kazemi. 'Road profile estimation using wavelet neural network and 7-DOF vehicle dynamic systems'. In: Journal of Mechanical Science and Technology 26.10 (2012), pp. 3029-3036.

[35] R. S. Barbosa. 'Vehicle dynamic response due to pavement roughness'. In: J. Brazilian Soc. Mech. Sci. Eng. XXXIII.3 (2011), pp. 302-307.

[36] R. S. Barbosa. 'Vehicle dynamic safety in measured rough pavement'. In: Journal of Transportation Engineering 137.5 (2011), pp. 305-310.

[37] R. S. Barbosa. 'Vehicle vibration response subjected to longwave measured pavement irregularity'. In: J. Mech. Eng. Autom. 2.2 (2012), pp. 17-24.

[38] K. De Zoysa, C. Keppitiyagama, G. P. Seneviratne et al. 'A public transport system based sensor network for road surface condition monitoring'. In: Proc. 2007 Work. Networked Syst. Dev. Reg. - NSDR '0\%. New York, USA: ACM Press, 2007.

[39] J. Eriksson, L. Girod, B. Hull et al. 'The pothole patrol: Using a Mobile Sensor Network for Road Surface Monitoring'. In: Proceeding 6th Int. Conf. Mob. Syst. Appl. Serv. - MobiSys '08. New York, USA: ACM Press, 2008.

[40] P. Mohan, V. N. Padmanabhan and R. Ramjee. 'Nericell: Rich Monitoring of Road and Traffic Conditions using Mobile Smartphones'. In: Proc. 6th ACM Conf. Embed. Netw. Sens. Syst. - SenSys '08. New York, USA: ACM Press, 2008. 
[41] M. Perttunen et al. 'Distributed Road Surface Condition Monitoring Using Mobile Phones'. In: Lect. Notes Comput. Sci. Ed. by C.-H. Hsu et al. Springer-Verlag Berlin Heidelberg, 2011, pp. 64-78.

[42] Y. Tai, C. Chan and J. Hsu. 'Automatic road anomaly detection using smart mobile device'. In: Conference on Artificial Intelligence and Applications (TAAI). Hsinchu, Taiwan, Nov. 2010.

[43] A. Mednis, G. Strazdins, R. Zviedris et al. 'Real time pothole detection using Android smartphones with accelerometers'. In: International Conference on Distributed Computing in Sensor Systems and Workshops (DCOSS). IEEE, June 2011, pp. 1-6.

[44] F. Carrera, S. Guerin and J. B. Thorp. 'BY THE PEOPLE, FOR THE PEOPLE: THE CROWDSOURCING OF "STREETBUMP": AN AUTOMATIC POTHOLE MAPPING APP'. In: ISPRS - Int. Arch. Photogramm. Remote Sens. Spat. Inf. Sci. XL-4/W1.4W1 (May 2013), pp. 19-23.

[45] M. Hoffmann, M. Mock and M. May. 'Road-quality classification and bump detection with bicycle-mounted smartphones'. In: UDM'13 Proc. 3rd Int. Conf. Ubiquitous Data Min. Vol. 1088. Beijing, China, 2013, pp. 39-43.

[46] H. Sharma, S. Naik, A. Jain et al. 'S-road assist: Road surface conditions and driving behavior analysis using smartphones'. In: 2015 International Conference on Connected Vehicles and Expo (ICCVE). IEEE, Oct. 2015, pp. 291-296.

[47] H.-w. Wang, C.-h. Chen, D.-y. Cheng et al. 'A Real-Time Pothole Detection Approach for Intelligent Transportation System'. In: Mathematical Problems in Engineering 2015 (2015), pp. 1-7.

[48] F. Seraj, B. J. van der Zwaag, A. Dilo et al. 'RoADS: A Road Pavement Monitoring System for Anomaly Detection Using Smart Phones'. In: Lect. Notes Comput. Sci. Ed. by M. Atzmueller et al. Vol. 9546. Lecture Notes in Computer Science. Springer International Publishing, 2016, pp. 128-146.

[49] H. P. M. and V. P. Gopi. 'Vehicle Vibration Signal Processing for Road Surface Monitoring'. In: IEEE Sens. J. 17.16 (Aug. 2017), pp. 5192-5197.

[50] A. Fox, B. V. Kumar, J. Chen et al. 'Multi-Lane Pothole Detection from Crowdsourced Undersampled Vehicle Sensor Data'. In: IEEE Trans. Mob. Comput. 16.12 (Dec. 2017), pp. 3417-3430.

[51] F. Cong, H. Hautakangas, J. Nieminen et al. 'Applying wavelet packet decomposition and one-class support vector machine on vehicle acceleration traces for road anomaly detection'. In: Lecture Notes in Computer Science 7951 LNCS.PART 1 (2013), pp. 291-299.

[52] Z. Li, I. Kolmanovsky, E. Atkins et al. 'Road anomaly estimation: Model based pothole detection'. In: 2015 Am. Control Conf. Vol. 2015-July. IEEE, July 2015, pp. 1315-1320. 
[53] S. Hegde, H. Mekali and G. Varaprasad. 'Pothole detection and inter vehicular communication'. In: 2014 IEEE Int. Conf. Veh. Electron. Saf. IEEE, Dec. 2014, pp. 84-87.

[54] R. Madli, S. Hebbar, P. Pattar et al. 'Automatic Detection and Notification of Potholes and Humps on Roads to Aid Drivers'. In: IEEE Sens. J. 15.8 (Aug. 2015), pp. $4313-4318$.

[55] Z. Zhang, X. Ai, C. K. Chan et al. 'An efficient algorithm for pothole detection using stereo vision'. In: 2014 IEEE Int. Conf. Acoust. Speech Signal Process. IEEE, May 2014, pp. 564-568.

[56] Y. Jo and S. Ryu. 'Pothole Detection System Using a Black-box Camera'. In: Sensors 15.12 (Nov. 2015), pp. 29316-29331.

[57] D.-W. Jang and R.-H. Park. 'Pothole detection using spatio-temporal saliency'. In: IET Intell. Transp. Syst. 10.9 (Nov. 2016), pp. 605-612.

[58] P. Wang, Y. Hu, Y. Dai et al. 'Asphalt Pavement Pothole Detection and Segmentation Based on Wavelet Energy Field'. In: Math. Probl. Eng. 2017 (2017), pp. 113.

[59] A. Tedeschi and F. Benedetto. 'A real-time automatic pavement crack and pothole recognition system for mobile Android-based devices'. In: Adv. Eng. Informatics 32 (Apr. 2017), pp. 11-25.

[60] B.-h. Kang and S.-i. Choi. 'Pothole detection system using 2D LiDAR and camera'. In: 2017 Ninth Int. Conf. Ubiquitous Futur. Networks. IEEE, July 2017, pp. 744746.

[61] M. W. Sayers and S. M. Karamihas. The little book of profiling. Michigan: University of Michigan, 1998.

[62] E. B. Spangler and W. J. Kelly. GMR road profilometer: a method for measuring road profile. Tech. rep. GMR-452. Warren, Michigan: General Motors Corporation Research Laboratories, 1964.

[63] R. La Barre, R. Forbes and S. Andrew. The Measurement and Analysis of Road Surface Roughness. Tech. rep. 1970/5. Nuneaton, Warwickshire (UK): MIRA (The Motor Industry Research Association), 1969.

[64] H. Gimmler, D. Ammon and J. Rauh. Road profiles: mobile measurement, data processing for efficient simulation and assessment of road properties. Tech. rep. VDI-Report No. 1912. Dusseldorf, Germany: VDI, 2005.

[65] J. Rauh and M. Mössner-Beigel. 'Tyre simulation challenges'. In: Vehicle System Dynamics 46.sup1 (2008), pp. 49-62.

[66] P. Andrén. 'Power spectral density approximations of longitudinal road profiles'. In: International Journal of Vehicle Design 40.1/2/3 (2006), pp. 2-14.

[67] V. Cossalter. Motorcycle Dynamics. 2nd ed. [place unknown]: LULU, 2006, p. 372. 
[68] M. Tanelli, M. Corno and S. M. Savaresi, eds. Modelling, simulation and control of two-wheeled vehicles. Chichester: Wiley, 2014, pp. 1-348.

[69] S. Suthaharan. Machine Learning Models and Algorithms for Big Data Classification. Vol. 36. Integrated Series in Information Systems. Boston, MA: Springer US, 2016, p. 364.

[70] S. B. Kotsiantis, I. D. Zaharakis and P. E. Pintelas. 'Machine learning: a review of classification and combining techniques'. In: Artif. Intell. Rev. 26.3 (Nov. 2006), pp. 159-190. 